\title{
Organic Semiconductors as Photoanodes for Solar-driven Photoelectrochemical Fuel Production
}

\author{
Arvindh Sekar and Kevin Sivula*
}

\begin{abstract}
The direct conversion of solar energy into chemical fuels, such as hydrogen, via photoelectrochemical (PEC) water splitting requires the efficient oxidation of water at a photoanode. While transition metal oxides have shown a significant success as photoanodes, their intrinsic limitations make them the bottleneck of PEC water splitting. Recently, initial research reports suggest that organic semiconductors (OSCs) could be possible alternative photoanode materials in both dye-sensitized and thin film photoelectrode configurations. Herein we review the progress to date, with a focus on the major issues faced by OSCs: stability and low photocurrent density in aqueous photoelectrochemical conditions. An outlook to the future of OSCs in photoelectrochemistry is also given.
\end{abstract}

Keywords: Conjugated polymers · Dye sensitized · Hydrogen · Oxygen evolution reaction · Water splitting

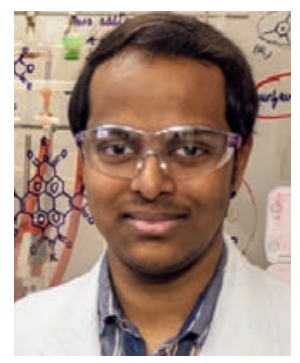

Arvindh Sekar is from Tamil Nadu in India and received a Bachelor degree in Chemical Science and Technology from Indian Institute of Technology Guwahati in 2015 and Master degree in Chemistry from the École polytechnique fédérale de Lausanne (EPFL) in 2017. He is currently pursuing his doctorate in EPFL in the group of Prof. Kevin Sivula. The current focus of his research is organic semiconductors used as photoanodes. In his free time, he enjoys reading novels and playing video games.

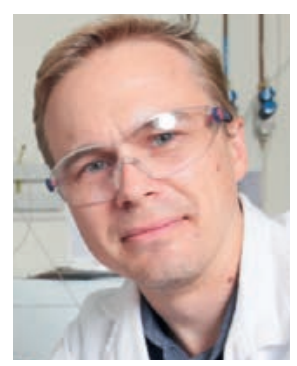

Kevin Sivula is an Associate Professor of Chemical Engineering and he leads the Laboratory for Molecular Engineering of Optoelectronic Nanomaterials (LIMNO) at EPFL while also teaching courses on Transport Phenomena, Chemical Engineering Practicals, Product design, and solar energy conversion systems.

\section{Introduction}

Converting our largest renewable energy source, solar energy, into the chemical energy of hydrogen (via water splitting), hydrocarbons (via $\mathrm{CO}_{2}$ reduction), and ammonia (via $\mathrm{N}_{2}$ reduction) has been the topic of immense importance since such chemical storage vectors, or solar fuels, solve the problem of solar energy intermittency and can provide feedstocks to the commodity chemicals industry. ${ }^{[1-3]}$ Of these, hydrogen is arguably the most attractive since there is abundant water on earth ${ }^{[4,5]}$ and hydrogen possesses a high gravimetric energy density. ${ }^{[6]}$ Indeed, a hydrogen-based energy economy is often imagined where fuel cells convert hydrogen into electrical power on demand for use in vehicles ${ }^{[7]}$ and residences. ${ }^{[8]}$ Accordingly, technologies for hydrogen storage ${ }^{[9-11]}$ and transportation ${ }^{[12]}$ are also under intense development.

To be economically viable, solar-driven hydrogen production needs to compete with current industrial methods of producing hydrogen: steam reforming of methane ${ }^{[13-15]}$ and anaerobic methane pyrolysis. ${ }^{[16,17]}$ While the significant reduction of greenhouse gas emission involved in solar-driven $\mathrm{H}_{2}$ production via water splitting is advantageous, it still essentially costs 4-5 times as much per kilogram of $\mathrm{H}_{2}$ produced compared to conventional methods. ${ }^{[18]}$ Hence there is an urgent need to advance solar driven water-splitting technologies to overcome this barrier. Broadly, the major types of solar-driven technologies to split water into $\mathrm{H}_{2}$ and $\mathrm{O}_{2}$ can be divided into three types: Photovoltaic-Electrolysis (PV-E), Photoelectrochemical (PEC), and Photocatalytic systems (PC). In this short review we briefly summarize these competing approaches, while motivating and highlighting the use of emerging organic (carbon-based) semiconductors to drive the watersplitting reactions. Subsequently, we focus on recent literature results that address a challenging aspect of employing organic semiconductors for solar-driven water splitting: using them as water oxidizing photoanodes.

\section{Approaches for Solar-driven Water Splitting}

Photovoltaic-Electrolysis (PV-E) technology simply couples available photovoltaic systems with water splitting electrolyzers. The photovoltaic component uses a semiconductor-based device to convert solar photons to excited electrons (driving an electrical current in an external circuit) and the electrolyzer device uses this electrical power to drive the two water-splitting half-reactions: water (or proton, $\mathrm{H}^{+}$) reduction on the cathode to form $\mathrm{H}_{2}$ and hydroxide (or water) oxidation on the anode to make $\mathrm{O}_{2}$. Effectively in PV-E, the photon-to-electron and electron-to-fuel conversions occur using different components that are electronically connected. Individually, as both technologies themselves are quite well established on a large scale, this method of producing hydrogen has been demonstrated with high efficiency ${ }^{[19,20]}$ and can be easily implemented at different scales. Unfortunately, as previously 
mentioned, the price of the $\mathrm{H}_{2}$ produced is not yet economically competitive with conventional $\mathrm{H}_{2}$ production techniques, so a common goal in research is to increase the solar-to-hydrogen (STH) efficiency of PV-E systems. However, systems with high STH efficiency require multiple junction PV-devices and concentrated solar irradiation, ${ }^{[21]}$ which are typically very expensive. As a benchmark example of a simple PV-E implementation under 1 sun illumination, the highest STH of $20.6 \%$ has been reported by Tae Nam and coworkers ${ }^{[22]}$ which used an AlGaAs as a PV device coupled to an electrolyzer (membrane electrode assembled configuration) with $\mathrm{IrO}_{x}$ as the oxygen evolution reaction catalyst (OERC) on the anode and Pt on nanocrystalline carbon as the hydrogen evolution reaction catalyst (HERC) on the cathode. About $78 \%$ of the electrical energy generated by the PV was effectively converted to hydrogen. Despite the progress with PV devices in general and the PV-E approach, many researchers look for an alternative approach that could produce $\mathrm{H}_{2}$ at a significantly reduced cost.

Photoelectrochemical (PEC) water splitting combines light harvesting and chemical fuel generation into a single component: the semiconductor (SC) photoelectrode. The SCs, which can either self-catalyze the water oxidation/reduction reactions directly using their surface or use electrocatalysts deposited at the SC-water interface, form the active part of the photoanode and photocathode in PEC water splitting. The advantages of this technology can be lower device complexity and potentially lower cost. Working photoelectrodes can easily be immersed in electrolytes to produce chemical fuels without any complicated electronic connections. The simplicity of the device architecture is visible from Fig. 1, where the scheme of a PEC photoanode/photocathode tandem cell is illustrated. Here the HERC/photocathode (SC1) and OERC/ photoanode (SC2) are wirelessly connected via an Ohmic contact and immersed in water-based electrolyte. SC1 and SC2 absorb complementary portions of the solar spectrum to build sufficient photopotential to drive the overall water-splitting reactions. The thermodynamic driving force for the electrolysis of water is 1.23 $\mathrm{eV}$ per molecule $\mathrm{H}_{2} \mathrm{O}$. In addition to the thermodynamic energy, accounting for energy losses due to charge recombination and catalytic overpotential of the materials, the valence band of the $\mathrm{SC}$ in the photoanode needs to be more positive than $1.23 \mathrm{~V}$ vs. the reversible hydrogen electrode $\left(\mathrm{V}_{\mathrm{RHE}}\right)$ and the conduction band of the photocathode needs to be more negative than $0 \mathrm{~V}_{\mathrm{RHE}}$. Upon illumination, the quasi Fermi level of minority charge carriers equilibrate, $E_{F, S C 2, p}^{*} \approx E_{F, S C 1, n}^{*}$ while the quasi fermi level of SC1 at the electrolyte/semiconductor interface is sufficient to transfer holes to OERC and oxidize water $\left(E_{F, S C 1, p}^{*}>E_{O_{2} / H_{2} O}^{o}\right)$ while the quasi fermi level of SC2 at the electrolyte/semiconductor inter- face is sufficient to transfer electrons to HERC and reduce water $\left(E_{F, S C 2, n}^{*}<E_{H^{+} / H_{2}}^{o}\right)$. The band bending in SC1 and SC2 results in an internal electric field which causes holes drifting towards OERC and electrons drifting towards HERC for overall water splitting.

In contrast to the electrochemically based systems described above, photocatalytic (PC) systems comprise the simplest of the three device architectures. SCs with appropriate energy band levels are suspended as micro- or nano-particles in water, and upon illumination the photogenerated electrons and holes are used by HERCs and OERCs on the surface of the particles or in solution to produce hydrogen and oxygen. The ease of this system configuration however comes with drawbacks. A lack of a driving force for charge carrier separation, poor STH efficiencies (around 1\%), and the requirement of sacrificial electron shuttles to afford overall water-splitting, to name a few, make this method of splitting water the least performant. ${ }^{[23]}$ However, from an economic perspective PC water splitting can potentially produce the most cost-effective $\mathrm{H}_{2}$ if the STH of the system can be raised to the level of the PEC or PV-E devices ( 10\%). Moreover, since the PC approach employs a direct SC/water interface like PEC water splitting, the further study of photoelectrodes with various semiconductors can help to gain insight into the limitations of the PC water splitting approach and further develop both of these systems towards viable commercial application.

Considering the SC materials that can be suitable for PEC or $\mathrm{PC}$ water splitting, the direct SC/water interface places harsh demands on the stability. The seminal report of PEC water splitting is often cited as Fujishima and Honda in 1972, ${ }^{[24]}$ where n-type $\mathrm{TiO}_{2}$, a wide energy gap, $\mathrm{E}_{\mathrm{g}}$, SC material was used as a photoanode to directly oxidize water on its surface under illumination. However, $\mathrm{TiO}_{2}$ cannot harness much of the incoming solar spectrum due to its wide $\mathrm{E}_{\mathrm{g}}$ of $3.1 \mathrm{eV}$. Similar wide $\mathrm{E}_{\mathrm{g}} \mathrm{SCs}_{\mathrm{sike}} \mathrm{SrTiO}_{3}$ $\left(\mathrm{E}_{\mathrm{g}}>3.2 \mathrm{eV}\right)$ and $\mathrm{KTaO}_{3}\left(\mathrm{E}_{\mathrm{g}}>3.6 \mathrm{eV}\right)$ have good relative performance based on the light that they can absorb, but they are limited to $\mathrm{STH}<2 \%$.[25,26]

More recently, smaller $\mathrm{E}_{\mathrm{g}} \mathrm{SC}$ materials in PC systems and as photocathodes/photoanodes capable of much higher STH, if used in a tandem cell, have been developed. A few examples of photocathodes with high performance include $\mathrm{p}$-Si based photocathodes with epitaxially grown $\mathrm{SrTiO}_{3}$ and a nanostructured HERC $(\mathrm{Pt})^{[27]}$ reaching a 1-Sun photocurrent density, $\mathrm{J}_{\mathrm{ph}}$, of $15 \mathrm{~mA} \mathrm{~cm}{ }^{-2}$ at $0 \mathrm{~V}_{\mathrm{RHE}}$ and inexpensive $\mathrm{Sb}_{2} \mathrm{Se}_{3}$ sublimed into compact, welloriented films protected by $\mathrm{TiO}_{2}$ and with $\mathrm{RuO}_{x}$ HERC deposited photoelectrochemically ${ }^{[28]}$ giving $\mathrm{J}_{\mathrm{ph}} \sim 30 \mathrm{~mA} \mathrm{~cm}^{-2}$ at $0 \mathrm{~V}_{\mathrm{RHE}}$.

While the photocathodes have almost reached the SC's theoretical maximum $\mathrm{J}_{\text {ph }}$, photoanodes however, typically perform poorer. The state of the art photoanode materials are metal oxides
Fig. 1. Scheme of a photoelectrochemical water-splitting tandem cell $E_{\mathrm{O}_{2} / \mathrm{H}_{2} \mathrm{O}}^{o}$ and $E_{\mathrm{H}^{+} / \mathrm{H}_{2}}^{o}$ refer to redox potentials for water oxidation and reduction, $E_{F, i, j}^{*}$ refers to quasi fermi levels of 'i' semiconductor for 'j' charge carrier. OERC and HERC are the water oxidation and reduction catalysts. Observe the electrons $\left(\mathrm{e}^{-}\right)$and holes $\left(\mathrm{h}^{+}\right)$generated on illumination accumulating at the HERC and OERC to split water.

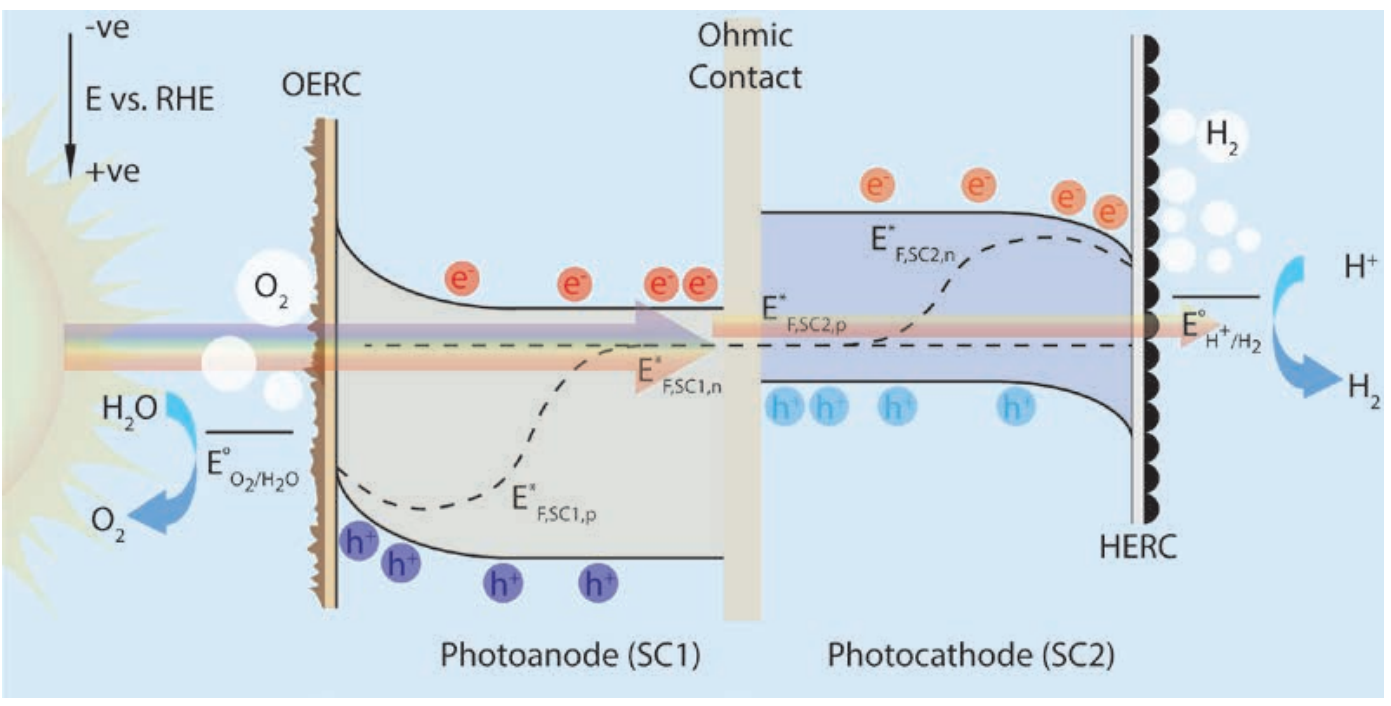


and oxynitrides. For example, $\mathrm{LaTiO}_{2} \mathrm{~N}^{[29]}$ gives (unstable) $\mathrm{J}_{\mathrm{ph}}$ of $8.9 \mathrm{~mA} \mathrm{~cm}^{-2}$ at $1.23 \mathrm{~V}_{\mathrm{RHE}}, \mathrm{BiVO}_{4}$ gives stable $\mathrm{J}_{\mathrm{ph}}$ of $4.0 \mathrm{~mA} \mathrm{~cm} \mathrm{~m}^{-2}$ at $0.6 \mathrm{~V}_{\mathrm{RHE}}$. These materials suffer from low $\mathrm{J}_{\mathrm{ph}}^{\mathrm{ph}}$ due to high $\mathrm{E}_{\mathrm{g}}$, crystallographic disorders, high internal recombination states due to traps, low absorption coefficients, low carrier hole mobilities and lifetimes of the SC. Moreover, these materials often require expensive processing techniques like vapor deposition which severely limit the scalability of the photoelectrode devices. ${ }^{[30-33]}$ Thus, there is a clear need for the development of new high performance photoanode materials.

Carbon-based organic semiconductors (OSCs) have been widely demonstrated in organic photovoltaics (OPVs) and dye sensitized solar cells (DSSCs), and have various advantages over the typical inorganic SCs used in photoanodes. Most of the OSCs used in OPVs and DSSCs are solution processable, which facilitates large area fabrication of devices using simple ink-based printing techniques while still obtaining high Power Conversion Efficiency (PCE) in photovoltaic devices. ${ }^{[34-37]}$ With relatively high charge mobilities and high light absorption properties, even thin films of OSCs lead to high PCEs (up to $17 \%$ in OPVs and $14 \%$ in DSSCs). ${ }^{[38-41]}$ Accordingly, the use of OSCs for solar-driven water splitting has been recently considered. OPVs coupled with electrolyzers as PV-E systems have been demonstrated, usually with multi-junction PV devices to obtain high open circuit potentials $(>1.23 \mathrm{~V})$. These devices require relatively expensive fabrication methods, and have scalability issues with respect to surface area of electrolyzer vs. solar cell. ${ }^{[42-44]}$ Alternatively, OSCs have been employed with a direct OSC/water interface in photocathodes for $\mathrm{H}_{2}$ production. For example, a donor:acceptor bulk heterojunction (BHJ) of PTB7-Th (donor) and PDI-V (acceptor) polymers (see chemical structures and photocathode schematic in Fig. 2) over $\mathrm{MoO}_{3}$ as the hole-selective transport layer (HTL $)^{[45]}$ and $\mathrm{RuO}_{x}$ as HERC resulted in a $\mathrm{J}_{\mathrm{ph}}$ of $8.2 \mathrm{~mA} \mathrm{~cm}^{-2}$ at $0 \mathrm{~V}_{\text {RHE }}$ retaining $85 \%$ of the initial $\mathrm{J}_{\mathrm{ph}}$ after 8 hours of operation.

While the use of OSCs as high performance and stable photocathodes have been demonstrated successfully in a number of recent reports, ${ }^{[45-50]}$ their use as photoanodes has been somewhat restrained. A major issue is likely the harsh oxidative electrochemical conditions. At such high applied potentials, the OSC needs to be functionally stable to oxidize water instead of decomposing. However, due to synthetic ease of tuning energy levels in OSCs, many OSCs are reported that possess suitable energy levels to oxidize water under illumination, and are able to produce $\mathrm{O}_{2}$ in the harsh environment of a photoanode, at least temporarily. In the next section we detail the device architectures that make functional photoanodes based on OSC and we report the progress made recently using OSCs as photoanodes for the water oxidation reaction. It is important to note that the OSC inherently only performs the light absorption, exciton generation (see section 3.2 below) and charge separation/transport processes. ${ }^{[51-53]}$ Unlike inorganic metal oxide photoanodes, the photogenerated hole at the OSC/water interface almost always requires the use of a water oxidation heterogeneous catalyst or a molecular catalyst (OERC) deposited on the photoanode to oxidize water, due to the kinetic complexity of the water oxidation reaction.

\section{Organic Semiconductor-based photoanodes}

Overall, there are two major strategies in which OSCs are used as photoanodes. The first borrows the device architecture from the DSSC and incorporates a mesoporous metal oxide layer over a conducting substrate onto which a monolayer of dye is sensitized. Light absorption by the dye leads to ultrafast injection of a photogenerated electron from the dye into the conduction band of the mesoporous metal oxide (typically $\mathrm{SnO}_{2}$ or $\mathrm{TiO}_{2}$ ), leaving the photogenerated hole on the dye and available to oxidize a species in the electrolyte. When these dye-sensitized photoelectrochemical cells (DS-PECs) are placed in contact with an aqueous electro-
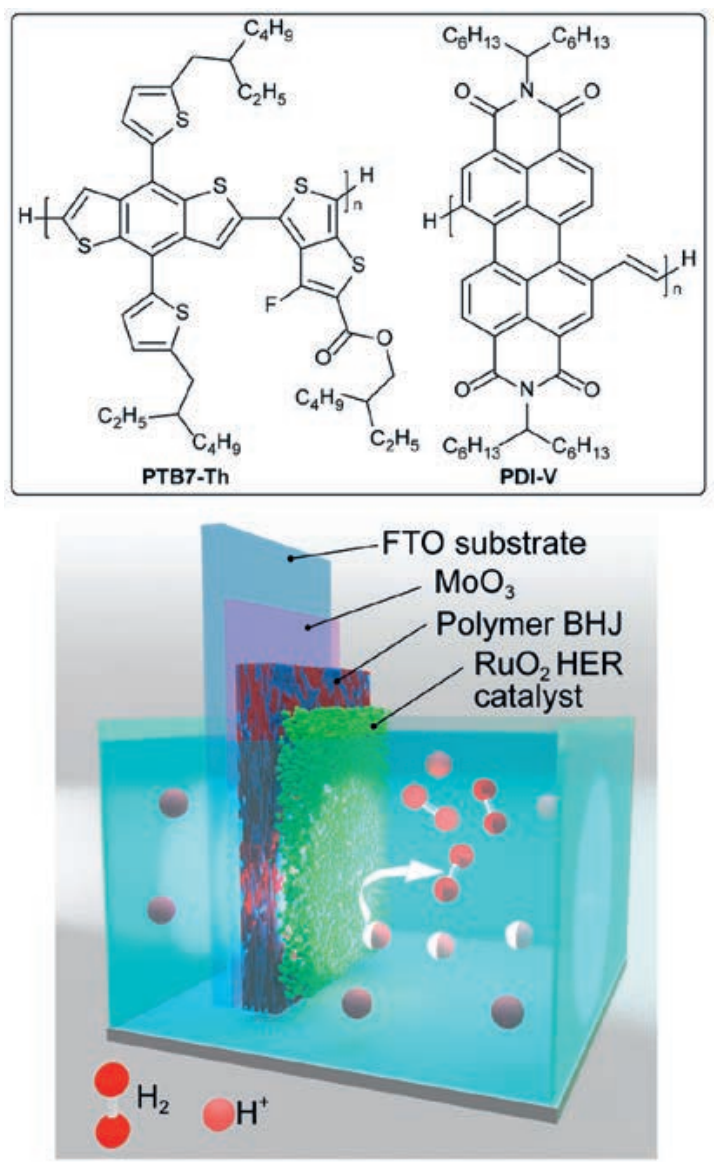

Fig. 2. Polymers used in a recently reported OSC-based photocathode (top). A schematic of the employed photocathode which employs a F-doped tin oxide (FTO) substrate, the polymers in a bulk heterojunction $(\mathrm{BHJ})$ and a direct contact of the OCSs/catalyst with the waterbased electrolyte (Bottom). Reproduced from ref. [45], copyright 2020 American Chemical Society.

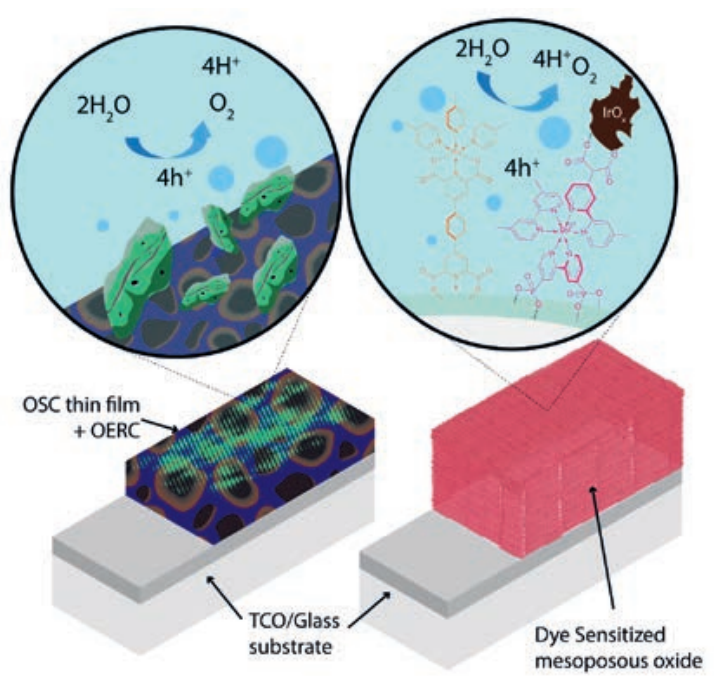

Fig. 3. Schematic diagram of TF-PEC (left) and DS-PEC (right). While the TF-PEC uses a heterogeneous OERC, in the DS-PEC, two ways of anchoring an OERC are shown. A molecular OERC (Ru complex in orange) is directly anchored on the metal oxide, or a heterogeneous OERC can be anchored via the dye (Ru complex in magenta).

lyte the hole can oxidize water if it can be transferred to an OERC first. In this case the requirement for the OSC to transport the photogenerated charge is reduced, since only a monolayer of light absorber is required. This allows the use of a wide variety of dyes. The second strategy employing OSCs in photoanodes involves 
replacing the dye sensitized mesoporous metal oxide with a thin film OSC, and can be called a thin film photoelectrochemical cell (TF-PEC). In this case the OSC acts both as a medium for light harvesting and photogenerated charge transport. Fig. 3 illustrates schematics of the two different photoanode architectures.

\subsection{Dye-sensitized Photoanodes}

Broadly, the DS-PECs can be further classified as those using metal-organic complexes as dye sensitizers (MC DS-PECs), and those which utilize chromophores without metal centers, i.e. 'metal-free' (MF DS-PECs). The metal-organic-complex-based dyes are inherently more expensive, based on raw materials, and likely more toxic. Hence MC DS-PECs could have limitations in terms of scalability and processability. [38] Synthetically, modifying the energy levels of these complexes is also limited by the substituents on the ligands, leading to constraints in terms of band gap and energy level tuning. ${ }^{52,54,55]}$ The demonstrated metal-organic complexes used in MC DS-PECs usually follow a similar molecular template: a ruthenium central ion ligated by aromatic
$\mathrm{N}$-heterocycles like bipyridines with anchoring groups like phosphonates is the primary photoabsorber (Fig. 4A shows dye molecular structures, which will be discussed below), and which is anchored to a thick (7-12 $\mu \mathrm{m})$ mesoporous electron accepting metal oxide scaffold. Reports of photoanodes using this concept and the other DS-PECs are summarized in Table 1 with typical performance parameters. ${ }^{[56-63]}$

Another known template for DS-PECs uses porphyrin derivatives as macromolecular dyes: both with metal centers, ${ }^{[64]}$ and as metal-free (sub)porphyrins. ${ }^{[65-68]}$ While these Ru-dyes and metal porphyrins are efficient photo-absorbers, their onset wavelength is limited to $<700 \mathrm{~nm}$, leading to limited light capture in the near infrared region of the solar spectrum. To extend the light harvesting, researchers have more recently employed alternate OSCs: robust intrinsic n-type OSCs like rylene diimide derivatives, ${ }^{699,70]}$ donor- $\pi$ conjugation-acceptor moieties $(D-\pi-A),{ }^{[71,72]}$ or metalfree (sub)porphyrins. ${ }^{[65,66,73]}$ Fig. 4B shows the structures of the $D-\pi$-A dyes employed in DS-PEC photoanodes where the incorporation of electronic push-pull phenomena at the molecular
Fig. 4. Subtypes of dyes in DSPECs: A) Ru- based dyes (red box), B) D- $\pi$-A dyes (blue box), C) porphyrin derivatives (green box), D) n-type OSC dyes (purple box, E) miscellaneous dyes (black box). Anchoring groups are bound to metal oxides.
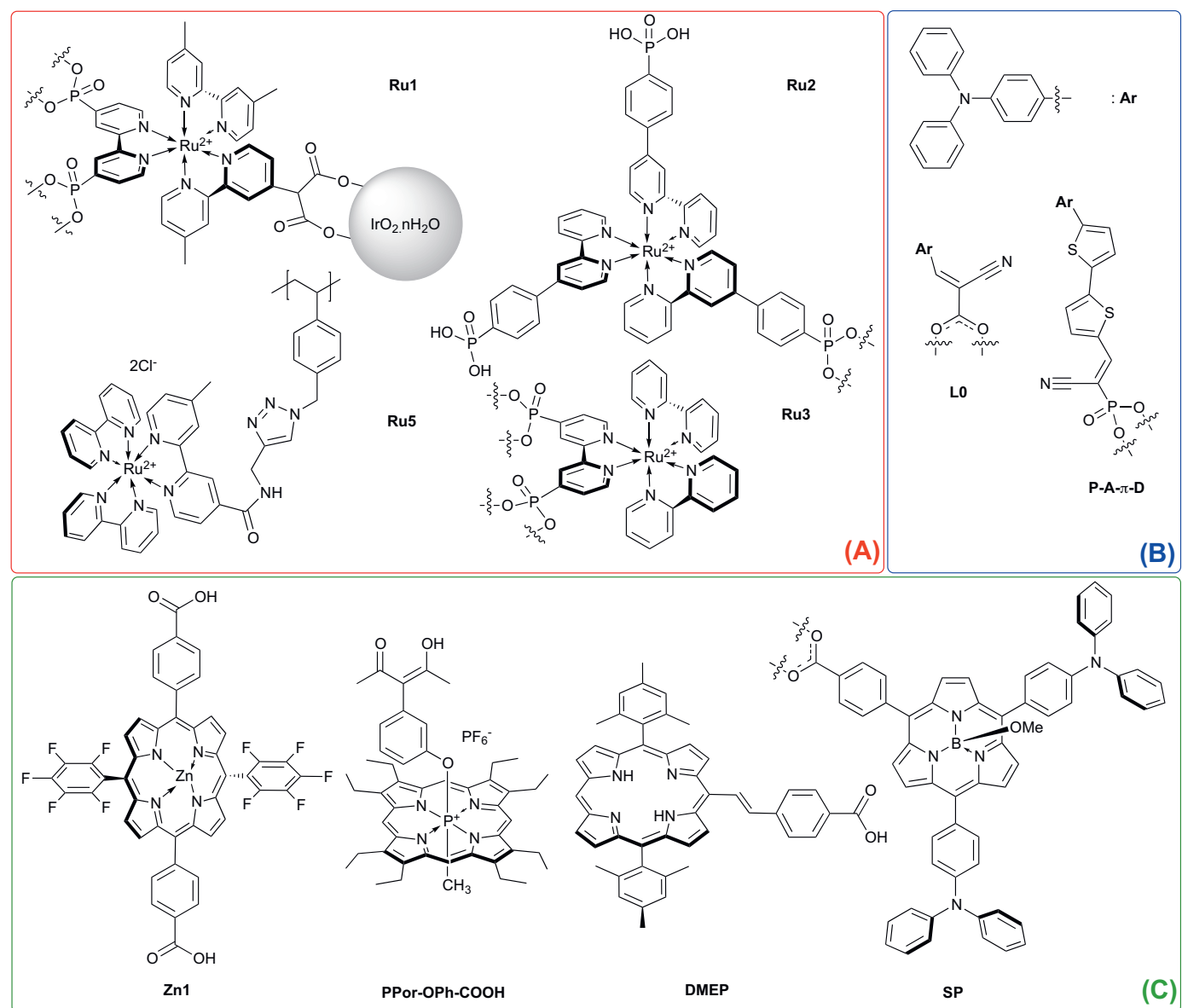

(B)
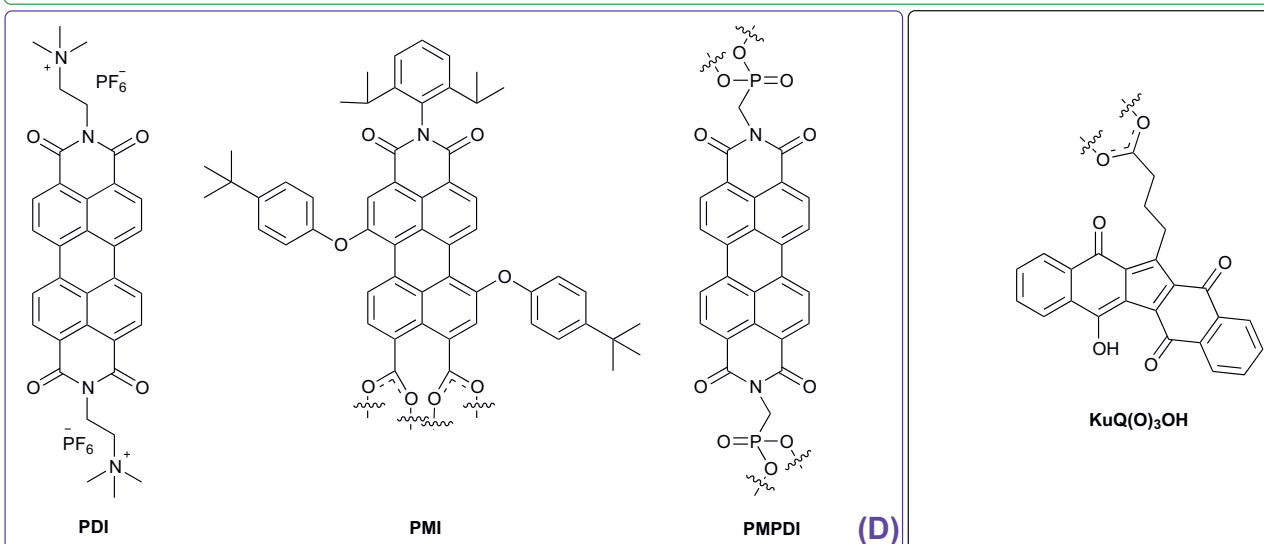

$\mathrm{KuQ}(\mathrm{O})_{3} \mathrm{OH}$
(C) 
Table 1. DS-PECs and operating parameters, observed $\mathrm{J}_{\mathrm{ph}}$ and stability with Faradaic efficiencies.

\begin{tabular}{|c|c|c|c|c|c|}
\hline $\begin{array}{l}\text { Overlayers (if any)/ OERC } \\
\text { and Photo-absorber }{ }^{\mathrm{a}} / \\
\text { Electron Transport Layer / } \\
\text { Electrode }\end{array}$ & $\begin{array}{l}\text { Illumination \& } \\
\text { Filters }(\lambda: \text { cutoff } \\
\text { wavelength) }\end{array}$ & $\begin{array}{l}\mathrm{J}_{\mathrm{ph}} / \mathrm{A} \mathrm{cm}^{-2} @ \\
\text { applied potential/ } \\
\mathrm{V}_{\mathrm{RHE}}\end{array}$ & $\begin{array}{l}\text { Stability } \\
\left(\mathrm{J}_{\mathrm{ph}} / \mathrm{A} \mathrm{cm} \mathrm{cm}^{-2}\right. \\
\text { after time/s })\end{array}$ & Electrolyte & $\begin{array}{l}\text { Faradaic } \\
\text { Efficiency for } \\
\mathrm{O}_{2} \text { generation } \\
/ \%\end{array}$ \\
\hline $\begin{array}{l}\mathrm{IrO}_{x} \cdot n \mathrm{H}_{2} \mathrm{O} / \mathbf{R u} \mathbf{1} / \\
\text { porous nanocrystalline } \\
\mathrm{TiO}_{2} \text { film }(9 \mu \mathrm{m}) / \\
\mathrm{FTO}^{[57]}\end{array}$ & $\lambda>410 \mathrm{~nm}$ & $\begin{array}{l}\sim 7.5 \times 10^{-7} \\
@ 0.54\end{array}$ & $\begin{array}{l}\sim 1.7 \times 10^{-7} \\
\text { after } 60\end{array}$ & $\begin{array}{l}\mathrm{Na}_{2} \mathrm{SiF}_{6-}^{-} \\
\mathrm{NaHCO}_{3} \text { buffer at } \\
\text { pH } 5.75 \\
0.5 \mathrm{M} \mathrm{Na}_{2} \mathrm{SO}_{4}\end{array}$ & - \\
\hline $\begin{array}{l}\text { IrWOC1 / Zn1/ } \\
\text { P25 } \mathrm{TiO}_{2} \text { nanoparticles film } \\
(10 \mu \mathrm{m}) / \mathrm{FTO}^{[64]}\end{array}$ & $\begin{array}{l}200 \mathrm{~mW} \cdot \mathrm{cm}^{-2} ; \lambda> \\
400 \mathrm{~nm}\end{array}$ & 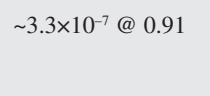 & $\begin{array}{l}\sim 3.0 \times 10^{-7} \\
\text { after } 60\end{array}$ & $\begin{array}{l}0.1 \mathrm{M} \mathrm{Na}_{2} \mathrm{SO}_{4} \text { at } \\
\text { pH } 7\end{array}$ & - \\
\hline $\begin{array}{l}\text { IrWOC2 + Ru2/ } \\
\mathrm{TiO}_{2} \text { film }(7 \mu \mathrm{m}) / \mathrm{FTO}\end{array}$ & $\lambda>410 \mathrm{~nm}$ & $1.8 \times 10^{-4} @ 0.54$ & $\begin{array}{l}\sim 9.0 \times 10^{-5} \\
\text { after } 95\end{array}$ & $\begin{array}{l}\mathrm{Na}_{2} \mathrm{SiF}_{6}-\mathrm{NaHCO}_{3} \\
\text { buffer at pH 5.8; } \\
0.1 \mathrm{M} \mathrm{LiClO}_{4}\end{array}$ & $\geq 85 \%$ \\
\hline $\begin{array}{l}\text { RuWOC1+Ru3/ } \\
\text { nano } \mathrm{TiO}_{2} \text { film } \\
(12 \mu \mathrm{m}) / \mathrm{FTO}^{[56]}\end{array}$ & $\begin{array}{l}300 \mathrm{~mW} \cdot \mathrm{cm}^{-2} \\
\lambda>400 \mathrm{~nm}\end{array}$ & $\sim 2.5 \times 10^{-3} @ 0.67$ & $\begin{array}{l}\sim 7.0 \times 10^{-4} \\
\text { after } 100\end{array}$ & $\begin{array}{l}\text { phosphate buffer } \\
\text { at pH } 6.8\end{array}$ & $83 \%$ \\
\hline $\begin{array}{l}\text { RuWOC2 } 2^{\text {b }}+\mathbf{R u} 3 / \\
\mathrm{TiO}_{2} \text { film } \\
(12 \mu \mathrm{m}) / \mathrm{FTO}^{[59]}\end{array}$ & $\begin{array}{l}300 \mathrm{~mW} \cdot \mathrm{cm}^{-2} \\
\lambda>400 \mathrm{~nm}\end{array}$ & $\sim 1.1 \times 10^{-3} @ 0.61$ & $\begin{array}{l}\sim 7.0 \times 10^{-4} \\
\text { after } 100\end{array}$ & $\begin{array}{l}0.1 \mathrm{M} \mathrm{Na}_{2} \mathrm{SO}_{4} \\
\text { at pH } 7\end{array}$ & $75 \%$ \\
\hline $\begin{array}{l}\mathrm{Al}_{2} \mathrm{O}_{3}(0.3 \mathrm{~nm}) / \mathbf{R u W O C 3}- \\
\mathrm{Ru}_{/} / \mathrm{TiO}_{2}(6.6 \mathrm{~nm}) / \mathrm{SnO}_{2}{ }^{\circ} / \\
\mathrm{FTO}^{[60]}\end{array}$ & $\begin{array}{l}455 \mathrm{~nm} \text { LED at } \\
46.2 \mathrm{~mW} / \mathrm{cm}^{2}\end{array}$ & $\begin{array}{l}\sim 5.0 \times 10^{-4} @ 0.6 \mathrm{~V} \\
\text { vs. } \mathrm{Pt}^{\mathrm{d}, \mathrm{e}}\end{array}$ & $\begin{array}{l}\sim 7.0 \times 10^{-5} \\
\text { after } \sim 1300\end{array}$ & $\begin{array}{l}0.1 \mathrm{M} \text { phosphate } \\
\text { buffer at } \mathrm{pH} 7\end{array}$ & $41 \%$ \\
\hline $\begin{array}{l}\mathrm{IrO}_{x} \cdot \mathrm{nH}_{2} \mathrm{O} / \mathrm{Ru} 3 / \mathrm{TiO}_{2}(6.6 \\
\mathrm{nm}) / \mathrm{nanoITO} \\
(3.2 \pm 0.5 \mu \mathrm{m}) / \mathrm{FTO}^{[61]}\end{array}$ & $\begin{array}{l}455 \mathrm{~nm} \text { LED } \\
\text { at } 14.5 \mathrm{~mW} / \mathrm{cm}^{2}\end{array}$ & $>6.0 \times 10^{-4} @ 0.61$ & $\begin{array}{l}1.1 \times 10^{-4} \\
\text { after } 7200\end{array}$ & $\begin{array}{l}\mathrm{Na}_{2} \mathrm{SiF}_{6}-\mathrm{NaHCO}_{3} \\
\text { buffer at pH 5.8; } \\
0.1 \mathrm{M} \mathrm{LiClO}_{4}\end{array}$ & - \\
\hline $\begin{array}{l}\text { RuWOC5-Zn2/ } \mathrm{TiO}_{2} \\
(12 \mu \mathrm{m}) / \mathrm{FTO}^{[109]}\end{array}$ & $\begin{array}{l}35 \mathrm{~mW} \cdot \mathrm{cm}^{-2} \\
\lambda>380 \mathrm{~nm}\end{array}$ & 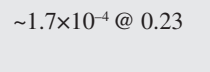 & $\begin{array}{l}7.0 \times 10^{-5} \\
\text { after } 60\end{array}$ & $\begin{array}{l}0.1 \mathrm{M} \text { phosphate } \\
\text { buffer at } \mathrm{pH} 7.3\end{array}$ & $33 \%$ \\
\hline $\begin{array}{l}\mathrm{CoWOC1}+\mathrm{Ru} 3 / \mathrm{TiO}_{2}^{\mathrm{c} /} \\
\text { FTO }^{[62]}\end{array}$ & $\begin{array}{l}100 \mathrm{~mW} \cdot \mathrm{cm}^{-2} \\
\lambda>400 \mathrm{~nm}\end{array}$ & $\sim 1.3 \times 10^{-4} @ 0.9$ & $\begin{array}{l}<4.0 \times 10^{-5} \\
\text { after } 120\end{array}$ & $\begin{array}{l}\text { phosphate buffer } \\
\text { at } \mathrm{pH} 6.8\end{array}$ & - \\
\hline $\begin{array}{l}(\mathrm{PA}-\mathrm{RuWOC3})_{5} \sim(\mathbf{R u 5})_{5}{ }_{5}^{\mathrm{f}} / \\
\mathrm{TiO}_{2}(6.6 \mathrm{~nm}) / \mathrm{SnO}_{2}{ }^{\mathrm{c}} / \mathrm{FTO}^{[63]}\end{array}$ & $\begin{array}{l}100 \mathrm{~mW} \cdot \mathrm{cm}^{-2} \\
\lambda>400 \mathrm{~nm}\end{array}$ & $\sim 1.8 \times 10^{-5} @ 0.85$ & $\begin{array}{l}\sim 6.0 \times 10^{-6} \\
\text { after } 600\end{array}$ & $\begin{array}{l}0.1 \mathrm{M} \text { phosphate } \\
\text { buffer at } \mathrm{pH} 7\end{array}$ & $22 \%$ \\
\hline $\begin{array}{l}\text { IrWOC1 + PPor-OPh- } \\
\text { COOH } / \mathrm{SnO}_{2} \text { film } \\
(10 \mu \mathrm{m}) / \mathrm{FTO}^{[73]}\end{array}$ & $\begin{array}{l}200 \mathrm{~mW} \cdot \mathrm{cm}^{-2} \\
\lambda>400 \mathrm{~nm}\end{array}$ & $\leq 2.0 \times 10^{-5} @ 0.91$ & $\begin{array}{l}\leq 2.0 \times 10^{-5} \\
\text { after } 300\end{array}$ & $\begin{array}{l}0.1 \mathrm{M} \mathrm{Na}_{2} \mathrm{SO}_{4} \\
\text { at pH } 7\end{array}$ & - \\
\hline $\begin{array}{l}\mathrm{IrO}_{\mathrm{x}}+\mathrm{DMEP}^{\mathrm{g} /} / \mathrm{TiO}_{2} \text { film } \\
(4 \mu \mathrm{m}) / \mathrm{FTO}^{[65]}\end{array}$ & $\lambda>410 \mathrm{~nm}$ & 8.0×10-5@0.70 & $\begin{array}{l}\sim 1.0 \times 10^{-5} \\
\text { after } 600\end{array}$ & $\begin{array}{l}0.1 \mathrm{M} \text { sodium } \\
\text { phosphate buffer at } \\
\text { pH } 6.8\end{array}$ & $102 \pm 5 \%$ \\
\hline $\begin{array}{l}\mathrm{IrO}_{2} / \mathrm{PDI} / \text { nanocrystalline } \\
\mathrm{WO}_{3}^{\text {h }} \text { film }(0.7 \mu \mathrm{m}) / \\
\mathrm{FTO}^{[69]}\end{array}$ & $\begin{array}{l}\text { AM } 1.5 \mathrm{G} \\
\lambda>435 \mathrm{~nm}\end{array}$ & $<7.0 \times 10^{-5} @ 0.77$ & $\begin{array}{l}\text { Chrono- } \\
\text { coulometry }\end{array}$ & $\begin{array}{l}0.1 \mathrm{M} \mathrm{NaClO}_{4} \\
\text { at } \mathrm{pH} 3\end{array}$ & - \\
\hline $\begin{array}{l}\text { RuWOC4 + Lo } / \mathrm{TiO}_{2} \\
(8 \mu \mathrm{m}) / \mathrm{FTO}^{[71]}\end{array}$ & $\begin{array}{l}\text { White LED at } 100 \\
\mathrm{~mW} \cdot \mathrm{cm}^{-2} ; \\
\lambda>400 \mathrm{~nm}\end{array}$ & 5.0×10-4@0.41 & $\begin{array}{l}\sim 2.0 \times 10^{-4} \\
\text { after } 400\end{array}$ & $\begin{array}{l}0.05 \mathrm{M} \text { phosphate } \\
\text { buffer at } \mathrm{pH} 7\end{array}$ & $73 \%$ \\
\hline $\begin{array}{l}\text { RuWOC6 + P-A- } \boldsymbol{\pi}-\mathbf{D} / \mathrm{TiO}_{2} \\
(3 \mathrm{~nm}) / \mathrm{SnO}_{2} \\
(8 \mu \mathrm{m}) / \mathrm{FTO}^{[72]}\end{array}$ & $\begin{array}{l}100 \mathrm{~mW} \cdot \mathrm{cm}^{-2} \\
\lambda>400 \mathrm{~nm}\end{array}$ & $\sim 1.4 \times 10^{-3} @ 0.85^{\mathrm{e}}$ & $\begin{array}{l}\sim 1.0 \times 10^{-4} \\
\text { after } 600\end{array}$ & $\begin{array}{l}0.1 \mathrm{M} \text { phosphate } \\
\text { buffer at pH 7;0.5 } \\
\mathrm{M} \mathrm{KNO}_{3}\end{array}$ & $8.2 \%$ \\
\hline $\begin{array}{l}\text { RuWOC4 + SP/ } \\
\mathrm{TiO}_{2} \text { film }(2 \mu \mathrm{m}) / \\
\text { FTO }^{[66]}\end{array}$ & $\begin{array}{l}\text { LED } \\
\text { at } 100 \mathrm{~mW} \cdot \mathrm{cm}^{-2} \\
\lambda>420 \mathrm{~nm}\end{array}$ & 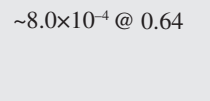 & $\begin{array}{l}\sim 6.0 \times 10^{-5} \\
\text { after } 30\end{array}$ & $0.1 \mathrm{M} \mathrm{NaF}$ at $\mathrm{pH} 7$ & $64 \%$ \\
\hline $\begin{array}{l}\text { IrWOC3 }{ }^{\mathrm{b}} / \mathrm{Al}_{2} \mathrm{O}_{3}(5 \mathrm{ALD} \\
\text { cycles }) / \mathbf{P M I} / \\
\mathrm{TiO}_{2}(10 \mathrm{~nm}) / \mathrm{FTO}^{[74]}\end{array}$ & $\begin{array}{l}200 \mathrm{~mW} \cdot \mathrm{cm}^{-2} \\
\lambda>410 \mathrm{~nm}\end{array}$ & 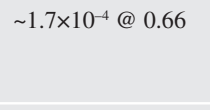 & $\begin{array}{l}<2.5 \times 10^{-5} \\
\text { after } 600\end{array}$ & $\begin{array}{l}1 \mathrm{M} \mathrm{Na}_{2} \mathrm{SO}_{4} \\
\text { at } \mathrm{pH} 2.5\end{array}$ & $22 \%$ \\
\hline $\begin{array}{l}\mathrm{CoO} / \text { PMPDI/ } \\
\mathrm{SnO}_{2}^{\mathrm{h}}(6 \mu \mathrm{m}) / \mathrm{FTO}^{[75]}\end{array}$ & $\begin{array}{l}\sim \mathrm{AM} 1.5 \mathrm{G} \\
\lambda>400 \mathrm{~nm}\end{array}$ & 2.0×10-5@1.41 & - & $\begin{array}{l}0.1 \mathrm{M} \text { phosphate } \\
\text { buffer at } \mathrm{pH} 7\end{array}$ & $31 \pm 7 \%$ \\
\hline $\begin{array}{l}\mathrm{Ru}_{4} \mathrm{POM}(1 \% \text { Nafion }) / \\
\mathrm{KuQ}(\mathbf{O})_{3} \mathrm{OH} / \mathrm{SnO}_{2} \\
(3 \mu \mathrm{m}) / \mathrm{FTO}^{[77]}\end{array}$ & $\begin{array}{l}\text { AM } 1.5 \mathrm{G} \\
\lambda>400 \mathrm{~nm}\end{array}$ & 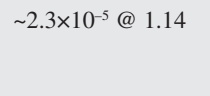 & $\begin{array}{l}\sim 3.0 \times 10^{-6} \\
\text { after } 5400\end{array}$ & $\begin{array}{l}0.1 \mathrm{M} \mathrm{Na}_{2} \mathrm{SiF}_{6}- \\
\mathrm{NaHCO}_{3} \text { buffer at } \\
\text { pH } 5.8\end{array}$ & $70 \pm 15 \%$ \\
\hline
\end{tabular}

aOER/dye refers to OERC bound to dye. OERC+dye refers to both co-sensitized on metal oxide. OERC-dye implies a binuclear bifunctional metal organic complex; 'Multiple OERCs investigated. Best performing molecule is mentioned; 'Thickness of mesoporous metal oxide not mentioned; 'Pt was used as both reference and counter electrode; e $J_{p H}$ here is photocurrent; the area of cell is not mentioned. ${ }^{\mathrm{f}}$ The polymer-OERC and polymeric Ru5 were deposited layer by layer 5 times over each other; 9Multiple dyes investigated. Best performing molecule is mentioned; ${ }^{\mathrm{h}} \mathrm{Mul}-$ tiple metal oxides investigated. Best performing oxide is mentioned; 'First $50 \mathrm{~s}$ of illumination cycle allows $1.25 \times 10^{-2} \mathrm{C} \mathrm{cm}^{-2}$, the third cycle allows $7.50 \times 10^{-2}$ $\mathrm{C} \mathrm{cm} \mathrm{cm}^{-2}$ level in their conjugation length can be noticed. In particular triphenyl amine-based dyes with cyanoacrylic acid anchoring groups have been successfully demonstrated in DS-PEC as photosensitizers. For example, in 2015, Sun and coworkers ${ }^{[71]}$ managed to make an organic tandem DS-PEC with both photocathode and photoanode sensitized by OSCs. The photoanode in use involved L0 dye (Fig. 4B) sensitized on $\mathrm{TiO}_{2}$ over fluorine-doped tin oxide (FTO) using RuWOC4 sensitized on $\mathrm{TiO}_{2}$ as the OERC (the structures of the OERCs used for DS-PECs are shown in Fig. 5). Slightly modifying this dye, Meyer and coworkers ${ }^{[72]}$ in 2016, used the P-A- $\pi$-D dye (Fig. 4B) on $\mathrm{TiO}_{2}$-coated $\mathrm{SnO}_{2}$ as the photoanode. While P-A- $\pi$-D-based photoanodes worked 
Fig. 5. Subtypes of OERCs: A) Ru molecular catalysts (blue box), B) Ir catalysts (red box), C) nonnoble metal catalyst (black box), D) bifunctional (photosensitizer + molecular OERC) binuclear metal complexes (green box).

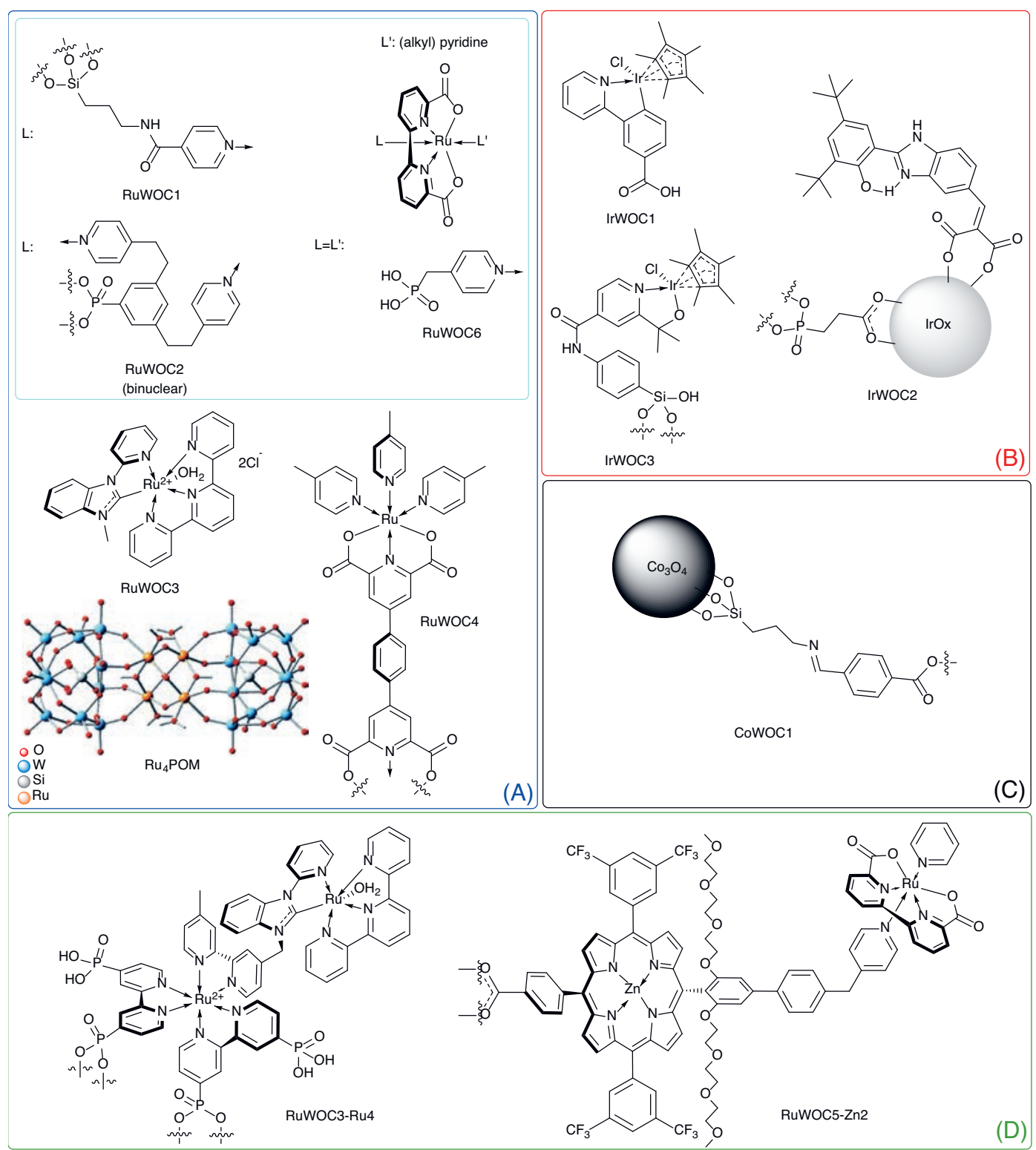

well in sacrificial conditions (20 $\mathrm{mM}$ hydroxyquinone, $\mathrm{H}_{2} \mathrm{Q}$ ), giving stable sacrificial $\mathrm{J}_{\text {phsac }}$ of $\sim 2.5 \mathrm{~mA} \mathrm{~cm}^{-2}$ at $0.66 \mathrm{~V}_{\mathrm{RHE}}$ in $\mathrm{pH}$ 7, with the OERC RuWOC6 (Fig. 5) in absence of sacrificial electron donors, $\mathrm{J}_{\mathrm{ph}}$ was significantly lower.

An interesting example of an n-type OSC dye is from Bignozzi and coworkers in 2015, who used an organic di-cation PDI (Fig. 4D) as the photoabsorber. ${ }^{[69]}$ In this study, multiple metal oxides like $\mathrm{TiO}_{2}, \mathrm{SnO}_{2}$ and $\mathrm{WO}_{3}$ were sensitized over long periods, when PDI was adsorbed on the nanocrystalline metal oxide. Spin-coated and air-dried $\mathrm{IrO}_{2}$ nanoparticles on PDI sensitized $\mathrm{WO}_{3}$ were used as the photoanode. Other examples of n-type OSC dyes include photoanodes by Wasielewski and coworkers ${ }^{[74]}$ and Finke and Kirner. ${ }^{[75]}$ PMI and PMPDI (Fig. 4D) were the dyes investigated, using IrWOC3 (Fig. 5) and $\mathrm{CoO}_{x}$ as catalysts respectively. Interestingly, Kirner and Finke also investigated their photoanodes with sacrificial electron donors (20 mM H $\left.\mathrm{H}_{2} \mathrm{Q}\right)$, reaching stable $\mathrm{J}_{\text {ph.sac }}$ of $1.1 \mathrm{~mA} \mathrm{~cm}^{-2}$ at $0.61 \mathrm{~V}_{\text {RHE }}$ in $\mathrm{pH} \mathrm{7.} \mathrm{Unfortunately,}$ with the OERC, $\mathrm{J}_{\mathrm{ph}}$ for water oxidation was in the $\mu \mathrm{A} \mathrm{cm}{ }^{-2}$ scale.

Examples of metal-free (sub)porphyrin photoanodes include PPor-OPh-COOH anchored to $\mathrm{SnO}_{2}$ and $\mathrm{SP}$ anchored to $\mathrm{TiO}_{2}$ (Fig. 4C) by van der Est and coworkers ${ }^{[73]}$ and Imahori and coworkers. ${ }^{[66]}$ With IrWOC3 and RuWOC4 as OERCs (Fig. 5) respectively, these photoanodes fared rather poorly in terms of performance and stability (Table 1). Indeed, stability is a criti- cal issue for the practical application of OSC-based photoanodes. From Table 1, it can be observed that engineering OERCs plays a major role in the device performance and stability. In all the cases, illumination was from the side of the back contact: F-doped $\mathrm{SnO}_{2}$ (FTO) or Sn-doped $\mathrm{In}_{2} \mathrm{O}_{3}$ (ITO), and the OERC was in contact with the electrolyte to oxidize water. Moreover, Mallouk and coworkers initially used hydrated $\mathrm{IrO}_{\mathrm{x}}$-bound to-dye-bound to- $\mathrm{TiO}_{2}$ as the photoanode in 2009[57] and in 2012, used ligand-modified $\mathrm{IrO}_{\mathrm{x}}\left(\mathrm{IrWOC} 2\right.$, Fig. 5) ${ }^{[58]}$ in which $\mathrm{J}_{\mathrm{ph}}$ marginally increased to 0.18 $\mathrm{mA} \cdot \mathrm{cm}^{-2}$ but was not very stable:[72,76] there was a $50 \%$ reduction in $\mathrm{J}_{\mathrm{ph}}$ after just 95 seconds of operation.

To increase $\mathrm{J}_{\mathrm{ph}}$ and improve stability molecular OERCs were considered. For example, the highest $\mathrm{J}_{\mathrm{ph}}$ in DS-PECs was obtained by Sun and coworkers in 2013-14, ${ }^{[56,59]}$ in near neutral pH conditions at $0.6-0.7 \mathrm{~V}_{\mathrm{RHE}}$. RuWOC1 (Fig. 5) bound to the $\mathrm{TiO}_{2}$ layer along with Ru3 resulted in $\mathrm{J}_{\mathrm{ph}} \sim 2.5 \mathrm{~mA} \cdot \mathrm{cm}^{-2}$, which dropped to $0.7 \mathrm{~mA} . \mathrm{cm}^{-2}$ after 100 seconds of operation. ${ }^{[56]}$ In another study, binuclear RuWOC2 (Fig. 5) with a phosphonate group anchored to $\mathrm{TiO}_{2}$ showed $\sim 83 \%$ higher $\mathrm{J}_{\mathrm{ph}}$ than its mononuclear variant, [59] however losing 37\% of its initial $\mathrm{J}_{\mathrm{ph}}$ within 100 seconds of operation. More recently in 2020, Sartorel and coworkers reported their DS-PEC photoanode which utilized a tetraruthenium polyoxometalate OERC $\left(\mathrm{Ru}_{4} \mathrm{POM}\right.$ with $1 \%$ Nafion, Fig. 5) showing oxygen evolution for 90 minutes, ${ }^{[77]}$ albeit with a modest $\mathrm{J}_{\mathrm{ph}}$. An 
overarching observation is that using ruthenium-based molecular catalysts always led to poor Faradaic efficiencies for oxygen evolution (from the lowest:[72] $8.2 \%$ to the highest:[56] 83\%), compared to heterogeneous oxide nanoparticles (lowest:[58] $\geq$ $85 \%$,highest: ${ }^{[65]} 102 \pm 5 \%$ ) and low stability (the best performing unprotected photoanode was operational for $<2$ minutes).

In addition to the OERC, another factor which affected the stability was the photosensitizer itself. It is hypothesized that in DSPECs, the loss of stability is primarily due to detachment of the dye from the metal oxide, ${ }^{[78,79]}$ or the oxidative decomposition of the chromophore. ${ }^{[72,76]}$ To address these issues, additional protective overlayers were used to enhance the stability. However, these overlayers are intrinsically insulating in nature for hole transport (e.g. $\mathrm{Al}_{2} \mathrm{O}_{3}{ }^{[60]}$ or polyacrylic acid, $\mathrm{PA}^{[63]}$ ) and therefore lower the $\mathrm{J}_{\mathrm{ph}}$. The longest functioning DS-PEC was demonstrated by Murray and coworkers ${ }^{[61]}$ in 2015. A thin layer of $\mathrm{TiO}_{2}$ by atomic layer deposition (ALD) over the mesoporous nanoITO ensured stable sorption of the dye and achieved the longest functional DSPEC with measured oxygen evolution for 2 hours of operation (with $\sim 80 \%$ loss in $\mathrm{J}_{\mathrm{ph}}$ ).

\subsection{Organic Semiconductor Thin Film Photoanodes}

DS-PECs in general suffer the same drawbacks as traditional DSSCs, such as charge recombination due to the high surface area of the $\mathrm{TiO}_{2} /$ dye interface. ${ }^{[80-82]}$ Moreover, the demonstrated stability of DS-PEC does not suggest that these photoanodes will be used in practical devices in the near future. These drawbacks warrant investigation into photoanodes with a different architecture to reduce charge recombination and improve the $\mathrm{J}_{\mathrm{ph}}$ and stability. Thin film (TF) based OSC PEC cells can be considered as an improvement as they reduce the device complexity. Indeed, given the relatively high charge carrier mobility in many OSCs, a reasonably-performing photoanode can be prepared by simply coating a thin film of an OSC on to a conductive substrate. However, while planar TFs of OSCs absorb light quite efficiently due to high absorption coefficients, they do not integrate a mechanism for charge carrier separation like the DS-PEC (via fast charge injection into the mesoporous oxide). In fact, given the different reorganization energies and dielectric constant of an OSC (compared to a typical inorganic semiconductor), coulombically bound electron-hole pairs (excitons) are typically present at room temperature and these need to be separated to create free charge carriers. Different strategies to enhance charge separation in thin film OSCs, compared to a simple monolayer TF-OSC are shown schematically in Fig. 6 and include multilayering of $\mathrm{OSCs}^{\left[{ }^{[3-86]}\right.}$ where different energy levels and intrinsic charge transport properties of the OSC layers can facilitate charge separation, and the blending of OSCs into $\mathrm{BHJs},{ }^{[87-90]}$ which increase the interfacial area between electron-donating (p-type) and electron-accepting (n-type) OSC phases. Both of these approaches create an internal photogenerated electric field which aids in exciton separation and transport of electrons and holes. In all cases, OERCs deposited on the surface or embedded in the OSC thin
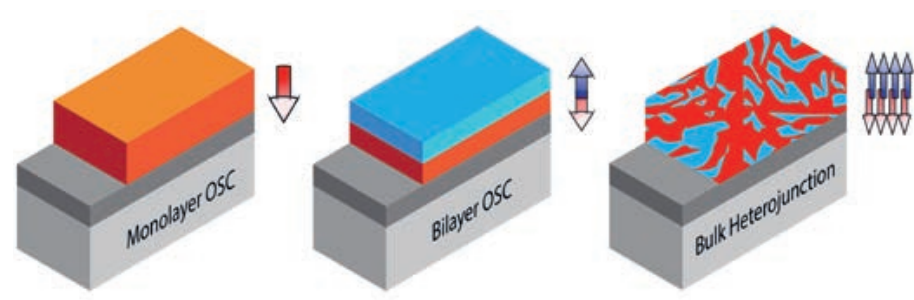

Fig. 6. A schematic of the three major subtypes of TF-PECs. n-type OSCs (in red), p-type OSCs (in blue) or both used together deposited on conducting substrates as photoanodes. Blue arrows indicate direction of hole transport, and red arrow indicate electron transport. films accept the photogenerated holes and oxidize water under operational conditions.

The earliest reported TF-PECs were by Norimatsu and coworkers, ${ }^{[11,92]}$ where the relatively insoluble but electrochemically robust OSCs PTCBI (n-type) and CoPc (p-type), see structures in Fig. 7, were successively vacuum deposited at high temperatures on indium tin oxide (ITO) substrates forming a bilayer photoanode. The use of an OERC (Nafion-covered $\mathrm{IrO}_{2}$ ) resulted in $\mathrm{J}_{\mathrm{ph}}$ of $10 \mu \mathrm{A} \mathrm{cm}^{-2}$ at $1.23 \mathrm{~V}_{\mathrm{RHE}}$. After this, Swiegers and coworkers used a Mn- molecular catalyst embedded in polymers $\left((\mathrm{TTh})_{\mathrm{n}}\right.$ and $\left.(\text { EDOT })_{\mathrm{n}}\right)$, see structures in Fig. 7, that were either electrochemically polymerized or vapor phase polymerized with the help of an oxidant on ITO or flexible ITO-polyethylene terephthalate (PET) substrates, ${ }^{[93,94]}$ which could oxidize seawater and were stable for 1 hour, albeit with $\mathrm{J}_{\mathrm{ph}} \sim 30 \mu \mathrm{A} \mathrm{cm} \mathrm{cm}^{-2}$ at potentials $>1.33 \mathrm{~V}_{\mathrm{RHE}}$.

Another class of TF-PECs focused on using traditional n-type OSCs as the photoabsorbers. For example, JinCai and coworkers used a hybrid trilayer photoanode of PCBM/ $\mathrm{PTCDA}_{11.6}: \mathrm{PCBM}_{1} / \mathrm{PTCDA}$ on ITO, ${ }^{[95]}$ achieving a $\mathrm{J}_{\mathrm{ph}}$ of $\sim 17$ $\mu \mathrm{A} \mathrm{cm}{ }^{-2}$ at $0.36 \mathrm{~V}_{\mathrm{RHE}}$ in acidic conditions. TF-PECs by Finke and coworkers ${ }^{[96]}$ used water-processed PMPDI spun coat on FTO (40-50 nm) with $\mathrm{CoO}_{\mathrm{x}}$ as the OERC. A J $\mathrm{J}_{\mathrm{ph}} \sim 0.28 \mathrm{~mA} \mathrm{~cm}^{-2}$ at $1.81 \mathrm{~V}_{\mathrm{RHE}}$ was achievable using this photoanode in neutral $\mathrm{pH}$ conditions. Another fullerene derivative $\left(\mathrm{PC}_{71} \mathrm{BM}\right)$ of a smaller bandgap with $\mathrm{ZnO}$ passivated overlayer showed photoanodic properties without any OERC. These photoanodes operated in alkaline conditions and were stable for over 1400 seconds, albeit with $\mathrm{J}_{\mathrm{ph}} \sim 15 \mu \mathrm{A} \mathrm{cm}{ }^{-2}$.

To improve the stability of TF-PEC anodes with OSC, our research group sought a robust organic semiconductor. BBL was known since the 1990s as a robust, semiconducting, stable ntype conjugated polymer. But due to poor solution processibility, it was not widely explored for photoelectrochemical applications. Bornoz et al. ${ }^{[97]}$ demonstrated an organic acid processed, spray-coated BBL film that was an extremely stable photoanode for water oxidation with Ni-Co OERC (where a thin, $1 \mathrm{~nm}, \mathrm{TiO}_{2}$ layer was used to help the adhesion between the OERC and the $\mathrm{BBL})$. In addition, by tuning the deposition method, this work established that increasing the surface area of the OSC/water interface helped to increase the rate of the solar-driven oxidation reaction. However, the most stable TF-PEC photoanodes to date used reduced graphene oxide (rGO) as an overlayer, with nanoNi or $\mathrm{NiOOH}$ as water oxidation catalyst, as demonstrated by Swiegers and coworkers ${ }^{[98]}$ and Chan Lim and coworkers ${ }^{[99]}$ respectively. While Chan Lim's photoanode consisted of an organic $\mathrm{BHJ}$ donor:acceptor ( $\left.\mathrm{P} 3 \mathrm{HT}: \mathrm{PC}_{61} \mathrm{BM}\right)$ blend as the photoactive layer, the photoanode of Swiegers aided the intrinsic electrocatalytic ability of Pt by depositing an OSC, (EDOT), over it. In alkaline $\mathrm{pH}$, these photoanodes effectively oxidized water and were stable for hours, and in the case of Chan Lim's work, at J $\sim 0.6 \mathrm{~mA} \mathrm{~cm}^{-2}$ with only 0.25 Sun illumination intensity. Results of TF-PEC photoanodes are summarized in Table 2. We note that this Table focuses on photoelectrodes and not 'buried junction' devices where the active semiconductors layers are not in close contact with the aqueous interface. However, results from these buried junction devices can also be impressive. Indeed, Jang and coworkers ${ }^{[100]}$ used a BHJ donor:acceptor blend in a fully functional OPV protected with overlayers and liquid metal eutectic interlayers, and demonstrated a stability for over 10 hours with about $10 \%$ loss in $\mathrm{J}_{\mathrm{ph}}$ when using a Ni-Fe layered double hydroxide OERC. A few other noteworthy publications also try other variations using similar metal-based and metal free OSCs but are not elaborated in this article due to poor stability, performance, oxygen evolution. ${ }^{[62,68,101]}$ Polyheptazine derivatives $\left(\mathrm{g}-\mathrm{C}_{3} \mathrm{~N}_{4}\right)$ also show decent performance and stability as photoanodes for water oxidation, ${ }^{[102-108]}$ however these semiconductors are not solution processable, hence are not elaborated here. 
Fig. 7. OSCs in TF-PECs: A) n-type (red box), B) p-type (blue box). Mn- catalyst is an OERC used.

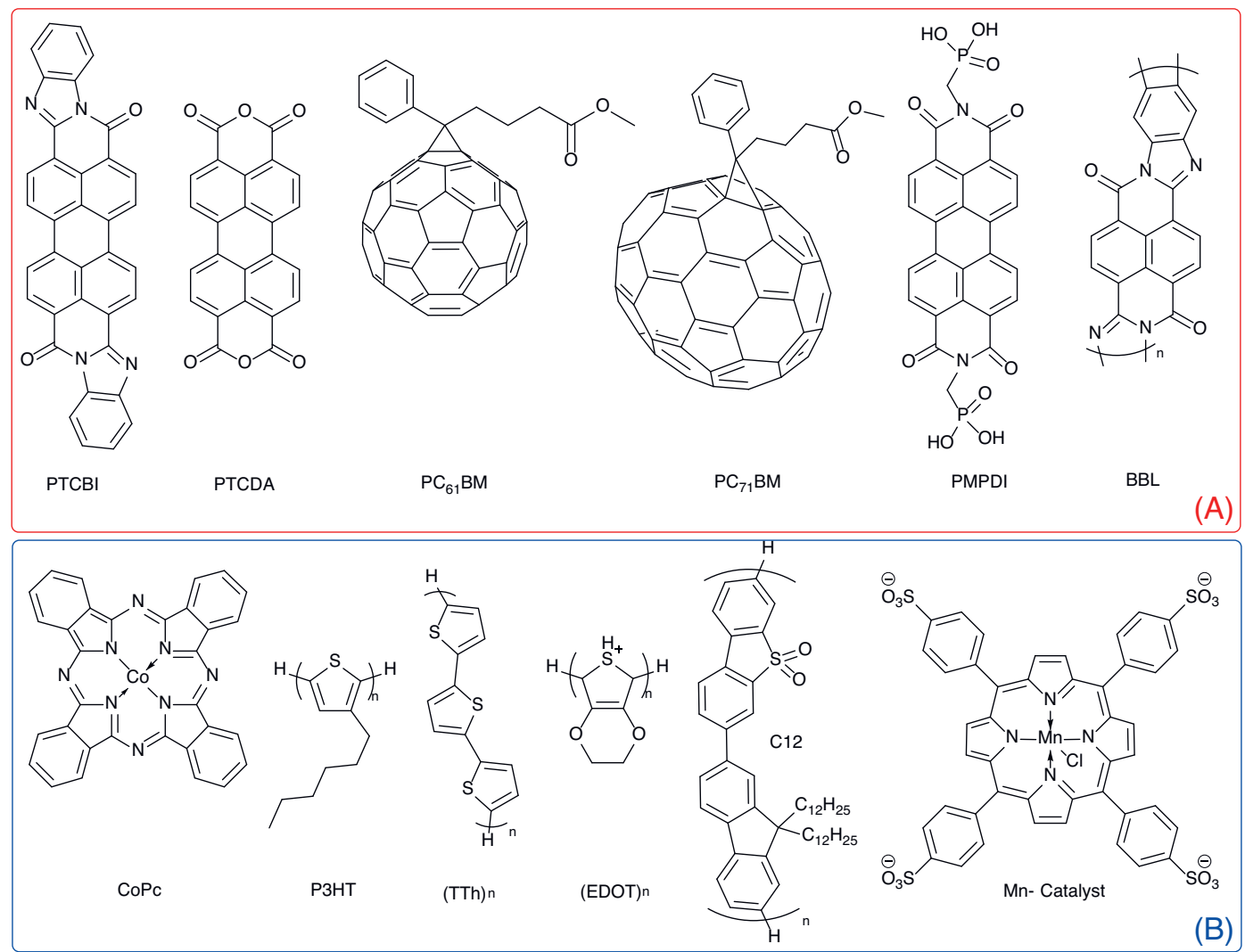

\section{Summary, Conclusions, and Outlook}

Taking together the recent results from using organic-based light harvesters for photoanodes in both the dye-sensitized and thin film approach, the utility of molecular engineering is apparent. The versatility of OSCs afforded by the tools of organic chemistry allows the fine tuning of structure to optimize light absorption and interfacial interactions (e.g. charge transfer to substrate or OERC). This versatility, together with the relatively mild electrochemical conditions of water reduction to $\mathrm{H}_{2}$, has allowed OSCs as photocathodes to reach high $\mathrm{J}_{\mathrm{ph}}$ values $\sim 10 \mathrm{~mA} \mathrm{~cm}^{-2}$, with stability in the hour scale. However, this performance has not yet been echoed for OSCs as photoanodes for solar driven water oxidation. Despite the numerous organic light harvesters and photoelectrode configurations already tested, solar photocurrents for water oxidation remain generally below $1 \mathrm{~mA} \mathrm{~cm}^{-2}$ with stability in the order of minutes. Research into OSC-based photoanodes, however, is at a nascent stage. During the first years of work, the field has addressed the challenges of preparing systems that have the appropriate energy levels and configurations to demonstrate that solar-driven water oxidation is possible from the thermodynamic and kinetic points of view. The next years of work must address the relatively low photocurrents and stability of OSCbased photoanodes. It is likely that both of these aspects can be improved by addressing the photogenerated charge accumulation in the OSC during photoanode operation. Indeed, it was recently pointed out that, for photocathodes, the accumulation of photogenerated charges in the OSC at the OSC/water interface drives the decomposition of the relatively fragile OSCs and decreases stability. ${ }^{[45]}$ Given the larger overpotentials required to drive the oxygen evolution reaction (due to its sluggish kinetics) compared to the hydrogen evolution reaction, it is reasonable to assume that photogenerated charge accumulation in a photoanode is more severe than in photocathodes. Accordingly, advanced strategies to rapidly extract charges (e.g. using hole transporting overlayers built from robust molecular components or inorganic species) are needed to prevent the oxidation of the light-absorbing OSCs. At the same time efforts are needed to develop OERCs with lower overpotentials for water oxidation. Alternatively, the direct molecular engineering of the organic semiconductor layers to drastically lower the HOMO and LUMO to increase the intrinsic robustness of these materials is also a possibility. As a drawback this can also move the onset potential for photocurrent to higher potentials, which makes implantation in an overall photoanode/photocathode tandem cell more challenging. Likely, the parallel advancement of all of these avenues is the way forward towards obtaining stable, high performance OSC photoanodes. Accomplishing this and subsequently demonstrating a highly efficient ( 10\% STH) and stable (lifetime of $\sim 1000$ hours) PEC tandem cell using an OSC-based photocathode and photoanode will represent a major step forward to inexpensive and direct solar hydrogen production.

\section{Acknowledgements}

We thank the Swiss National Science Foundation (project number 200021_169215) for partial financial support of this work.

Received: December 3, 2020

[1] D. Gust, T. A. Moore, A. L. Moore, Acc. Chem. Res. 2009, 42, 1890, https://doi.org/10.1021/ar900209b

[2] W. Haije, H. Geerlings, Environ. Sci. Technol. 2011, 45, 8609, https://doi.org/10.1021/es203160k.

[3] N. S. Lewis, Science 2016, 351, https://doi.org/10.1126/science.aad1920.

[4] A. Heller, Science 1984, 223, 1141, https://doi.org/10.1126/science.223.4641.1141.

[5] M. Grätzel, Nature 2001, 414, 338 .

[6] N. P. Brandon, Z. Kurban, Philos. Trans. A 2017, 375, https://doi.org/10.1098/rsta.2016.0400.

[7] Y. Manoharan, S. E. Hosseini, B. Butler, H. Alzhahrani, B. T. F. Senior, T. Ashuri, J. Krohn, Appl. Sci. 2019, 9, 2296, https://doi.org/10.3390/app9112296.

[8] P. E. Dodds, I. Staffell, A. D. Hawkes, F. Li, P. Grünewald, W. McDowall, P. Ekins, Int. J. Hydrog. Energy 2015, 40, 2065, https://doi.org/10.1016/j.ijhydene.2014.11.059.

[9] M. Ni, Energy Explor. Exploit. 2006, 24, 197 , https://doi.org/10.1260/014459806779367455.

[10] J. Andersson, S. Grönkvist, Int. J. Hydrog. Energy 2019, 44, 11901, https://doi.org/10.1016/j.ijhydene.2019.03.063. 
Table 2. TF-PECs and operating parameters, observed Jph and stability with Faradaic efficiencies.

\begin{tabular}{|c|c|c|c|c|c|}
\hline $\begin{array}{l}\text { Overlayers (if any)/ OERC } \\
\text { and Photo-absorber/ } \\
\text { Electron Transport Layer / } \\
\text { Electrode }\end{array}$ & $\begin{array}{l}\text { Illumination \& } \\
\text { Filters } \\
\text { ( } \lambda \text { : cutoff } \\
\text { wavelength) }\end{array}$ & $\begin{array}{l}\mathrm{J}_{\mathrm{ph}} / \mathrm{A} \mathrm{cm}^{-2} @ \\
\text { applied potential } \\
/ \mathrm{V}_{\mathrm{RHE}}\end{array}$ & $\begin{array}{l}\text { Stability } \\
\left(\mathrm{J}_{\mathrm{ph}} / \mathrm{A} \mathrm{cm}^{-2}\right. \\
\text { after time } \\
\text { / s })\end{array}$ & Electrolyte & $\begin{array}{l}\text { Faradaic } \\
\text { Efficiency for } \\
\mathrm{O}_{2} \text { generation } \\
/ \%\end{array}$ \\
\hline $\begin{array}{l}\text { CoPc }(0.19 \mu \mathrm{m}) / \text { PTCBI }(0.6 \\
\mu \mathrm{m}) / \mathrm{ITO}^{[91]}\end{array}$ & $\begin{array}{l}70 \\
\mathrm{~mW} \cdot \mathrm{cm}^{-2}\end{array}$ & 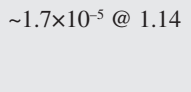 & $\begin{array}{l}\text { Stable at } \\
\sim 5.0 \times 10^{-6} \\
\text { for } \sim 3600\end{array}$ & $\begin{array}{l}\mathrm{NaOH} \text { (aq.) at } \mathrm{pH} \\
11^{\mathrm{a}, \mathrm{b}}\end{array}$ & - \\
\hline $\begin{array}{l}\text { Nafion }(30 \mathrm{~nm})-\mathrm{IrO}_{2} / \mathbf{H}_{2} \mathbf{P c} \\
(0.16 \mu \mathrm{m}) / \mathbf{P T C B I}(0.45 \\
\mu \mathrm{m}) / \mathrm{ITO}^{[92]}\end{array}$ & $\begin{array}{l}70 \\
\mathrm{~mW} \cdot \mathrm{cm}^{-2}\end{array}$ & $\sim 1.0 \times 10^{-5} @ 1.23$ & $\begin{array}{l}\sim 5.0 \times 10^{-6} \\
\text { after } 60\end{array}$ & $\begin{array}{l}\mathrm{KOH}(\text { aq. }) \text { at } \mathrm{pH} \\
10^{\mathrm{a}}\end{array}$ & - \\
\hline $\begin{array}{l}(\text { TTh })_{3 \mathrm{n}}-(\text { Mn catalyst })_{n} / \\
\text { ITO }^{[93]}\end{array}$ & $\begin{array}{l}50 \mathrm{~W} \text { halogen } \\
\text { lamp }\end{array}$ & $\sim 2.3 \times 10^{-5} @ 1.51$ & $\begin{array}{l}\text { Stable over } \\
24 \text { hours }\end{array}$ & $\begin{array}{l}0.1 \mathrm{M} \mathrm{Na}_{2} \mathrm{SO}_{4} \text { at } \\
\text { pH } 7\end{array}$ & $\begin{array}{l}>99.2 \% \text { in } \\
\text { seawater }\end{array}$ \\
\hline 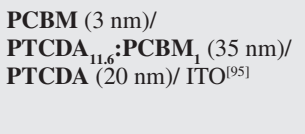 & $\begin{array}{l}110 \mathrm{~mW} \cdot \mathrm{cm}^{-2} ; \\
\lambda>420 \mathrm{~nm}\end{array}$ & $\sim 1.7 \times 10^{-4} @ 0.36$ & $\begin{array}{l}<1.0 \times 10^{-4} \\
\text { after } 1000\end{array}$ & $\begin{array}{l}35 \mathrm{mM} \mathrm{KNO}_{3} \\
\text { at pH } 2.0 \text { (adjusted } \\
\text { with } 1 \mathrm{M} \mathrm{H}_{2} \mathrm{SO}_{4} \text { ) }\end{array}$ & $\sim 92 \%$ \\
\hline 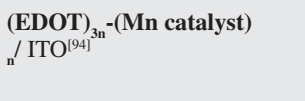 & $\begin{array}{l}>3 \text { sun intensity; } \\
\lambda>420 \mathrm{~nm}\end{array}$ & 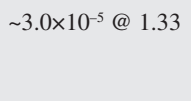 & $\begin{array}{l}\sim 2.6 \times 10^{-5} \\
\text { after } 3600\end{array}$ & Seawater at $\mathrm{pH} 8.57$ & $\begin{array}{l}>99.2 \% \text { in } \\
\text { seawater }\end{array}$ \\
\hline $\begin{array}{l}\mathrm{CoO}_{\chi}^{/} \text {PMPDI }(40-50 \mathrm{~nm}) / \\
\text { ITO }^{[96]}\end{array}$ & $100 \mathrm{~mW} \cdot \mathrm{cm}^{-2}$ & 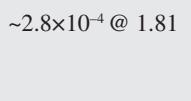 & $\begin{array}{l}\sim 1.0 \times 10^{-4} \\
\text { after } 300\end{array}$ & $\begin{array}{l}0.1 \mathrm{M} \text { potassium } \\
\text { phosphate buffer } \\
\text { at } \mathrm{pH} 7\end{array}$ & $80 \pm 15 \%$ \\
\hline $\begin{array}{l}\mathrm{Ni}-\mathrm{Co} / \mathrm{TiO}_{2}(1 \mathrm{~nm}) / \\
\mathbf{B B L}(230 \mathrm{~nm}) / \mathrm{FTO}^{[97]}\end{array}$ & $100 \mathrm{~mW} \cdot \mathrm{cm}^{-2}$ & 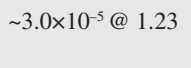 & $\begin{array}{l}\text { Stable at } \\
1.03 \times 10^{-5}\end{array}$ & $\begin{array}{l}\text { Phosphate buffer at } \\
\mathrm{pH} 7^{\mathrm{d}}\end{array}$ & $82 \pm 16 \%$ \\
\hline $\begin{array}{l}\text { rGO/ NiO-OH/ }(\text { EDOT) } \\
\text { n:PSS/ P3HT:PC }{ }_{61} \text { BM }(1: 1 ; \\
100 \mathrm{~nm}) / \mathrm{ZnO}_{\text {ITO }} \text { ITO] }^{[99]}\end{array}$ & $\begin{array}{l}100 \mathrm{~mW} \cdot \mathrm{cm}^{-2} \\
\text { AM } 1.5 \mathrm{G}\end{array}$ & $>1.0 \times 10^{-4} @ 1.23$ & $\begin{array}{l}\text { Stable at } \\
\sim 6.0 \times 10^{-5} \\
\text { after } 4 \mathrm{hr}\end{array}$ & $\begin{array}{l}0.1 \mathrm{M} \mathrm{Na}_{2} \mathrm{SO}_{4} \text { at } \\
\mathrm{pH} \sim 13.0\end{array}$ & - \\
\hline $\begin{array}{l}\mathrm{ZnO}(<2 \mathrm{~nm}) / \mathbf{P C}_{71} \mathbf{B M}(\sim 60 \\
\mathrm{nm}) / \mathrm{ITO}^{[110]}\end{array}$ & $\begin{array}{l}100 \mathrm{~mW} \cdot \mathrm{cm}^{-2} \\
\text { AM } 1.5 \mathrm{G}\end{array}$ & $<1.5 \times 10^{-5} @ 1.23$ & $\begin{array}{l}<6 \times 10^{-6} \text { after } \\
1400\end{array}$ & $\begin{array}{l}0.1 \mathrm{M} \mathrm{KOH} \\
\text { at } \mathrm{pH} \sim 13\end{array}$ & $\sim 33 \%$ \\
\hline $\begin{array}{l}\text { rGO/nano-Ni/ } \\
\text { (EDOT) }_{\mathbf{n}} / \mathrm{Pt} / \mathrm{FTO}^{[98]}\end{array}$ & 0.25 sun intensity & $5.5 \times 10^{-4} @ 1.7$ & $\begin{array}{l}6.0 \times 10^{-4} \\
\text { after } 50 \mathrm{hr}\end{array}$ & $\begin{array}{l}0.2 \mathrm{M} \mathrm{Na}_{2} \mathrm{SO}_{4} \text { at } \\
\text { pH } 12\end{array}$ & $>99.9 \%$ \\
\hline $\begin{array}{l}\mathrm{C12} / \mathrm{TiO}_{2} \text { nanorods }(250 \\
\mathrm{nm}) / \mathrm{FTO}^{[111]}\end{array}$ & $\begin{array}{l}\text { Xe lamp; } \\
\lambda>420 \mathrm{~nm}\end{array}$ & 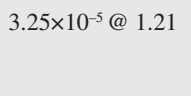 & $\begin{array}{l}>3.0 \times 10^{-5} \\
\text { after } 200\end{array}$ & $\begin{array}{l}0.5 \mathrm{M} \mathrm{Na}_{2} \mathrm{SO}_{4} \text { at } \\
\mathrm{pH} \sim 7.0\end{array}$ & - \\
\hline
\end{tabular}

aUnder argon atmosphere; 'bacrificial electron shuttle of $1 \mathrm{mM}$ TEA was used; ' $\mathrm{Cl}_{2}$ detection in $\mathrm{O}_{2}$ gas by Merckoquant ${ }^{\circledR}$ test strips; ${ }^{\mathrm{d}} 0.5 \mathrm{M} \mathrm{Na}_{2} \mathrm{SO}_{4}+0.09 \mathrm{M} \mathrm{KH}_{2} \mathrm{PO}_{4}+$ $0.01 \mathrm{M} \mathrm{K}_{2} \mathrm{HPO}_{4}$
[11] E. Rivard, M. Trudeau, K. Zaghib, Materials 2019, 12, https://doi.org/10.3390/ma12121973.

[12] X. J. Li, J. D. Allen, J. A. Stager, A. Y. Ku, Clean Energy 2020, 4, 26, https://doi.org/10.1093/ce/zkz033.

[13] L. Kaiwen, Y. Bin, Z. Tao, Energy Sources Part B 2018, 13, 109, https://doi.org/10.1080/15567249.2017.1387619.

[14] A. Bahadori, T. Kashiwao, Pet. Sci. Technol. 2019, 37, 1425 https://doi.org/10.1080/10916466.2019.1587466.

[15] G. Franchi, M. Capocelli, M. De Falco, V. Piemonte, D. Barba, Membranes 2020, 10, https://doi.org/10.3390/membranes 10010010

[16] S. Schneider, S. Bajohr, F. Graf, T. Kolb, ChemBioEng Rev. 2020, 7, 150, https://doi.org/10.1002/cben.202000014.

[17] G. A. V. Wald, M. S. Masnadi, D. C. Upham, A. R. Brandt, Sustain. Energy Fuels 2020, 4, 4598, https://doi.org/10.1039/D0SE00427H.

[18] P. Nikolaidis, A. Poullikkas, Renew. Sust. Energ. Rev. 2017, 67, 597, https://doi.org/10.1016/j.rser.2016.09.044.

[19] S. Koumi Ngoh, D. Njomo, Renew. Sust. Energ. Rev. 2012, 16, 6782, https://doi.org/10.1016/j.rser.2012.07.027.

[20] N. A. Kelly, T. L. Gibson, D. B. Ouwerkerk, Int. J. Hydrog. Energy 2008, 33, 2747, https://doi.org/10.1016/j.ijhydene.2008.03.036.

[21] J. H. Kim, D. Hansora, P. Sharma, J.-W. Jang, J. S. Lee, Chem. Soc. Rev. 2019, 48, 1908, https://doi.org/10.1039/C8CS00699G.

[22] W. J. Chang, K.-H. Lee, H. Ha, K. Jin, G. Kim, S.-T. Hwang, H. Lee, S.-W Ahn, W. Yoon, H. Seo, J. S. Hong, Y. K. Go, J.-I. Ha, K. T. Nam, ACS Omega 2017, 2, 1009, https://doi.org/10.1021/acsomega.7b00012.

[23] Q. Wang, K. Domen, Chem. Rev. 2020, 120, 919 https://doi.org/10.1021/acs.chemrev.9b00201.

[24] A. Fujishima, K. Honda, Nature 1972, 238,37, https://doi.org/10.1038/238037a0.

[25] T. Watanabe, A. Fujishima, K. Honda, Bull. Chem. Soc. Jpn. 1976, 49, 355 , https://doi.org/10.1246/bcsj.49.355.
[26] I. E. Paulauskas, J. E. Katz, G. E. J. Jr, N. S. Lewis, L. A. Boatner, G. M. Brown, J. Electrochem. Soc. 2009, 156, B580, https://doi.org/10.1149/1.3089281.

27] L. Ji, M. D. McDaniel, S. Wang, A. B. Posadas, X. Li, H. Huang, J. C. Lee, A. A. Demkov, A. J. Bard, J. G. Ekerdt, E. T. Yu, Nat. Nanotechnol. 2015, 10, 84, https://doi.org/10.1038/nnano.2014.277.

[28] W. Yang, J. H. Kim, O. S. Hutter, L. J. Phillips, J. Tan, J. Park, H. Lee, J. D. Major, J. S. Lee, J. Moon, Nat. Commun. 2020, 11, https://doi.org/10.1038/s41467-020-14704-3.

[29] S. Akiyama, M. Nakabayashi, N. Shibata, T. Minegishi, Y. Asakura, M. Abdulla-Al-Mamun, T. Hisatomi, H. Nishiyama, M. Katayama, T. Yamada, K. Domen, Small 2016, 12, 5468, https://doi.org/https://doi.org/10.1002/smll.201601929.

[30] A. R. Bielinski, S. Lee, J. J. Brancho, S. L. Esarey, A. J. Gayle, E. Kazyak, K. Sun, B. M. Bartlett, N. P. Dasgupta, Chem. Mater. 2019, 31, 3221, https://doi.org/10.1021/acs.chemmater.9b00065.

[31] Y. W. Chen, J. D. Prange, S. Dühnen, Y. Park, M. Gunji, C. E. D. Chidsey, P. C. McIntyre, Nat. Mater. 2011, 10, 539, https://doi.org/10.1038/nmat3047.

[32] I. A. Cordova, Q. Peng, I. L. Ferrall, A. J. Rieth, P. G. Hoertz, J. T. Glass, Nanoscale 2015, 7, 8584, https://doi.org/10.1039/C4NR07377K.

[33] M. F. Lichterman, A. I. Carim, M. T. McDowell, S. Hu, H. B. Gray, B. S. Brunschwig, N. S. Lewis, Energy Environ. Sci. 2014, 7, 3334 , https://doi.org/10.1039/C4EE01914H

[34] H.-P. Jen, M.-H. Lin, L.-L. Li, H.-P. Wu, W.-K. Huang, P.-J Cheng, E. W.-G. Diau, ACS Appl. Mater. Interfaces 2013, 5, 10098, https://doi.org/10.1021/am402687j.

[35] A. Y. Al-Ahmad, F. Almayhi, M. F. Al-Mudhaffer, M. J. Griffith, W. Liu, S. Li, K. Sivunova, D. Elkington, N. A. Cooling, K. Feron, M. Shi, W. Belcher, H. Chen, P. Dastoor, T. R. Andersen, Sustain. Energy Fuels 2020, 4, 940, https://doi.org/10.1039/C9SE01149H. 
[36] K. Y. Mitra, A. Alalawe, S. Voigt, C. Boeffel, R. R. Baumann, Micromachines 2018, 9, https://doi.org/10.3390/mi9120642

[37] B. Fan, W. Zhong, L. Ying, D. Zhang, M. Li, Y. Lin, R. Xia, F. Liu, H.-L. Yip, N. Li, Y. Ma, C. J. Brabec, F. Huang, Y. Cao, Nat. Commun. 2019, 10, 4100, https://doi.org/10.1038/s41467-019-12132-6.

[38] Y. Cui, H. Yao, L. Hong, T. Zhang, Y. Tang, B. Lin, K. Xian, B. Gao, C. An, P. Bi, W. Ma, J. Hou, Natl. Sci. Rev. 2020, 7, 1239, https://doi.org/10.1093/nsr/nwz200.

[39] Y. Cui, H. Yao, J. Zhang, T. Zhang, Y. Wang, L. Hong, K. Xian, B. Xu, S. Zhang, J. Peng, Z. Wei, F. Gao, J. Hou, Nat. Commun. 2019, 10, 2515, https://doi.org/10.1038/s41467-019-10351-5.

[40] Y. Lin, B. Adilbekova, Y. Firdaus, E. Yengel, H. Faber, M. Sajjad, X. Zheng, E. Yarali, A. Seitkhan, O. M. Bakr, A. El-Labban, U. Schwingenschlögl, V. Tung, I. McCulloch, F. Laquai, T. D. Anthopoulos, Adv. Mater. 2019, 31, 1902965, https://doi.org/10.1002/adma.201902965.

[41] K. Kakiage, Y. Aoyama, T. Yano, K. Oya, J. Fujisawa, M. Hanaya, Chem. Commun. 2015, 51, 15894, https://doi.org/10.1039/C5CC06759F.

[42] X. Elias, Q. Liu, C. Gimbert-Suriñach, R. Matheu, P. Mantilla-Perez, A Martinez-Otero, X. Sala, J. Martorell, A. Llobet, ACS Catal. 2016, 6, 3310, https://doi.org/10.1021/acscatal.6b01036.

[43] Y. Gao, V. M. L. Corre, A. Gaïtis, M. Neophytou, M. A. Hamid, K. Takanabe, P. M. Beaujuge, Adv. Mater. 2016, 28, 3366, https://doi.org/10.1002/adma.201504633.

[44] S. Esiner, G. W. P. van Pruissen, M. M. Wienk, R. A. J. Janssen, J. Mater. Chem. A 2016, 4, 5107, https://doi.org/10.1039/C5TA10459A

[45] L. Yao, N. Guijarro, F. Boudoire, Y. Liu, A. Rahmanudin, R. A. Wells, A Sekar, H.-H. Cho, J.-H. Yum, F. Le Formal, K. Sivula, J. Am. Chem. Soc. 2020, 142, 7795, https://doi.org/10.1021/jacs.0c00126.

[46] F. Fumagalli, S. Bellani, M. Schreier, S. Leonardi, H. C. Rojas, A Ghadirzadeh, G. Tullii, A. Savoini, G. Marra, L. Meda, M. Grätzel, G. Lanzani, M. T. Mayer, M. R. Antognazza, F. D. Fonzo, J. Mater. Chem. A 2016, 4, 2178, https://doi.org/10.1039/C5TA09330A.

[47] L. Francàs, E. Burns, L. Steier, H. Cha, L. Solà-Hernández, X. Li, P. S. Tuladhar, R. Bofill, J. García-Antón, X. Sala, J. R. Durrant, Chem. Commun. 2018, 54, 5732, https://doi.org/10.1039/C8CC01736K

[48] A. Mezzetti, F. Fumagalli, A. Alfano, D. Iadicicco, M. R. Antognazza, F. di Fonzo, Faraday Discuss. 2017, 198, 433, https://doi.org/10.1039/c6fd00216a.

[49] L. Steier, S. Bellani, H. C. Rojas, L. Pan, M. Laitinen, T. Sajavaara, F. D. Fonzo, M. Grätzel, M. R. Antognazza, M. T. Mayer, Sustain. Energy Fuels 2017, 1, 1915, https://doi.org/10.1039/C7SE00421D.

[50] H. C. Rojas, S. Bellani, F. Fumagalli, G. Tullii, S. Leonardi, M. T. Mayer, M. Schreier, M. Grätzel, G. Lanzani, F. D. Fonzo, M. R. Antognazza, Energy Environ. Sci. 2016, 9, 3710, https://doi.org/10.1039/C6EE01655C.

[51] B. M. Savoie, N. E. Jackson, L. X. Chen, T. J. Marks, M. A. Ratner, Acc. Chem. Res. 2014, 47, 3385, https://doi.org/10.1021/ar5000852.

[52] A. Pallikkara, K. Ramakrishnan, Int. J. Energy Res. 2020, https://doi.org/10.1002/er.5941.

[53] W. Cao, J. Xue, Energy Environ. Sci. 2014, 7, 2123, https://doi.org/10.1039/C4EE00260A

[54] I. M. Abdellah, A. El-Shafei, RSC Adv. 2020, 10, 27940, https://doi.org/10.1039/D0RA03916K.

[55] P. Brogdon, H. Cheema, J. H. Delcamp, ChemSusChem 2018, 11, 86, https://doi.org/https://doi.org/10.1002/cssc.201701441.

[56] Y. Gao, X. Ding, J. Liu, L. Wang, Z. Lu, L. Li, L. Sun, J. Am. Chem. Soc. 2013, 135, 4219, https://doi.org/10.1021/ja400402d.

[57] W. J. Youngblood, S.-H. A. Lee, Y. Kobayashi, E. A. Hernandez-Pagan, P. G. Hoertz, T. A. Moore, A. L. Moore, D. Gust, T. E. Mallouk, J. Am. Chem. Soc. 2009, 131, 926, https://doi.org/10.1021/ja809108y.

[58] Y. Zhao, J. R. Swierk, J. D. Megiatto, B. Sherman, W. J. Youngblood, D. Qin, D. M. Lentz, A. L. Moore, T. A. Moore, D. Gust, T. E. Mallouk, Proc. Natl. Acad. Sci. USA 2012, 109, 15612, https://doi.org/10.1073/pnas.1118339109.

[59] Zhang Linlin, Gao Yan, Ding Xin, Yu Ze, Sun Licheng, ChemSusChem 2014, 7, 2801, https://doi.org/10.1002/cssc.201402561.

[60] L. Alibabaei, B. D. Sherman, M. R. Norris, M. K. Brennaman, T. J. Meyer, Proc. Natl. Acad. Sci. USA 2015, 112, 5899, https://doi.org/10.1073/pnas.1506111112.

[61] K. E. Michaux, A. A. Gambardella, L. Alibabaei, D. L. Ashford, B. D. Sherman, R. A. Binstead, T. J. Meyer, R. W. Murray, J. Phys. Chem. C 2015, 119, 17023, https://doi.org/10.1021/acs.jpcc.5b05711.

[62] P. Wei, B. Hu, L. Zhou, T. Su, Y. Na, J. Energy Chem. 2016, 25, 345, https://doi.org/10.1016/j.jechem.2016.03.020.

[63] G. Leem, B. D. Sherman, A. J. Burnett, Z. A. Morseth, K.-R. Wee, J. M. Papanikolas, T. J. Meyer, K. S. Schanze, ACS Energy Lett. 2016, 1, 339, https://doi.org/10.1021/acsenergylett.6b00171.

[64] G. F. Moore, J. D. Blakemore, R. L. Milot, J. F. Hull, H. Song, L. Cai, C. A. Schmuttenmaer, R. H. Crabtree, G. W. Brudvig, Energy Environ. Sci. 2011, 4, 2389, https://doi.org/10.1039/C1EE01037A.

[65] J. R. Swierk, D. D. Méndez-Hernández, N. S. McCool, P. Liddell, Y. Terazono, I. Pahk, J. J. Tomlin, N. V. Oster, T. A. Moore, A. L. Moore, D. Gust, T. E. Mallouk, Proc. Natl. Acad. Sci. USA 2015, 112, 1681, https://doi.org/10.1073/pnas.1414901112.
[66] M. Yamamoto, Y. Nishizawa, P. Chábera, F. Li, T. Pascher, V. Sundström, L. Sun, H. Imahori, Chem. Commun. 2016, 52, 13702 , https://doi.org/10.1039/C6CC07877J.

[67] A. Nayak, R. R. Knauf, K. Hanson, L. Alibabaei, J. J. Concepcion, D. L. Ashford, J. L. Dempsey, T. J. Meyer, Chem. Sci. 2014, 5, 3115, https://doi.org/10.1039/C4SC00875H.

[68] A. Nayak, S. Roy, B. D. Sherman, L. Alibabaei, A. M. Lapides, M. K. Brennaman, K.-R. Wee, T. J. Meyer, ACS Appl. Mater. Interfaces 2016, 8 , 3853, https://doi.org/10.1021/acsami.5b10587.

[69] F. Ronconi, Z. Syrgiannis, A. Bonasera, M. Prato, R. Argazzi, S. Caramori, V. Cristino, C. A. Bignozzi, J. Am. Chem. Soc. 2015, 137, 4630, https://doi.org/10.1021/jacs.5b01519.

[70] R. J. Lindquist, B. T. Phelan, A. Reynal, E. A. Margulies, L. E. Shoer, J. R. Durrant, M. R. Wasielewski, J. Mater. Chem. A 2016, 4, 2880, https://doi.org/10.1039/C5TA05790F

[71] F. Li, K. Fan, B. Xu, E. Gabrielsson, Q. Daniel, L. Li, L. Sun, J. Am. Chem. Soc. 2015, 137, 9153, https://doi.org/10.1021/jacs.5b04856.

[72] K.-R. Wee, B. D. Sherman, M. K. Brennaman, M. V. Sheridan, A. Nayak, L. Alibabaei, T. J. Meyer, J. Mater. Chem. A 2016, 4, 2969, https://doi.org/10.1039/C5TA06678F

[73] P. K. Poddutoori, J. M. Thomsen, R. L. Milot, S. W. Sheehan, C. F. A. Negre, V. K. R. Garapati, C. A. Schmuttenmaer, V. S. Batista, G. W. Brudvig, A. van der Est, J. Mater. Chem. A 2015, 3, 3868, https://doi.org/10.1039/C4TA07018F

[74] R. J. Kamire, K. L. Materna, W. L. Hoffeditz, B. T. Phelan, J. M. Thomsen, O. K. Farha, J. T. Hupp, G. W. Brudvig, M. R. Wasielewski, J. Phys. Chem. C 2017, 121, 3752, https://doi.org/10.1021/acs.jpcc.6b11672.

[75] J. T. Kirner, R. G. Finke, ACS Appl. Mater. Interfaces 2017, 9, 27625, https://doi.org/10.1021/acsami.7b05874.

[76] D. L. Ashford, B. D. Sherman, R. A. Binstead, J. L. Templeton, T. J. Meyer, Angew. Chem. Int. Ed. Engl. 2015, 54, 4778 , https://doi.org/https://doi.org/10.1002/anie.201410944.

[77] G. A. Volpato, M. Marasi, T. Gobbato, F. Valentini, F. Sabuzi, V. Gagliardi, A. Bonetto, A. Marcomini, S. Berardi, V. Conte, M. Bonchio, S. Caramori, P. Galloni, A. Sartorel, Chem. Commun. 2020, 56, 2248 , https://doi.org/10.1039/C9CC09805D.

[78] H.-J. Son, C. Prasittichai, J. E. Mondloch, L. Luo, J. Wu, D. W. Kim, O. K. Farha, J. T. Hupp, J. Am. Chem. Soc. 2013, 135, 11529, https://doi.org/10.1021/ja406538a.

[79] C. L. Gray, P. Xu, A. J. Rothenberger, S. J. Koehler, E. Elacqua, B. H. Milosavljevic, T. E. Mallouk, J. Phys. Chem. C 2020, 124, 3542, https://doi.org/10.1021/acs.jpcc.0c00493.

[80] N. F. M. Sharif, M. Z. A. A. Kadir, S. Shafie, S. A. Rashid, W. Z. Wan Hasan, S. Shaban, Results Phys. 2019, 13, 102171, https://doi.org/10.1016/j.rinp.2019.102171.

[81] S. A. Haque, E. Palomares, B. M. Cho, A. N. M. Green, N. Hirata, D. R. Klug, J. R. Durrant, J. Am. Chem. Soc. 2005, 127, 3456 , https://doi.org/10.1021/ja0460357.

[82] E. Palomares, J. N. Clifford, S. A. Haque, T. Lutz, J. R. Durrant, Chem. Commun. 2002, 1464, https://doi.org/10.1039/B202515A.

[83] S. Saleh Ardestani, R. Ajeian, M. Nakhaee Badrabadi, M. Tavakkoli, Solar Energy Mater. Solar Cells 2013, 111, 107, https://doi.org/10.1016/j.solmat.2012.12.039.

[84] J. Lee, Y. K. Jung, D. Y. Lee, J.-W. Jang, S. Cho, S Son, J. Jeong, S. H. Park, Synth. Metals 2015, 199, 408, https://doi.org/10.1016/j.synthmet.2014.12.013.

[85] K. H. Lee, P. E. Schwenn, A. R. G. Smith, H. Cavaye, P. E. Shaw, M. James, K. B. Krueger, I. R. Gentle, P. Meredith, P. L. Burn, Adv. Mater. 2011, 23 766, https://doi.org/10.1002/adma.201003545.

[86] S. Dong, K. Zhang, B. Xie, J. Xiao, H.-L. Yip, H. Yan, F. Huang, Y. Cao, Adv. Energy Mater. 2019, 9, 1802832, https://doi.org/10.1002/aenm.201802832.

[87] D. A. Vithanage, A. Devižis, V. Abramavičius, Y. Infahsaeng, D. Abramavičius, R. C. I. MacKenzie, P. E. Keivanidis, A. Yartsev, D. Hertel, J. Nelson, V. Sundström, V. Gulbinas, Nat. Commun. 2013, 4, 2334, https://doi.org/10.1038/ncomms3334.

[88] R. Zhou, Z. Jiang, C. Yang, J. Yu, J. Feng, M. A. Adil, D. Deng, W. Zou, J. Zhang, K. Lu, W. Ma, F. Gao, Z. Wei, Nat. Commun. 2019, 10, 5393 , https://doi.org/10.1038/s41467-019-13292-1.

[89] M. C. Heiber, A. A. Herzing, L. J. Richter, D. M. DeLongchamp, J. Mater Chem. C 2020, 8, 15339, https://doi.org/10.1039/D0TC03087B.

[90] A. J. Mozer, N. S. Sariciftci, L. Lutsen, D. Vanderzande, R. Österbacka, M. Westerling, G. Juška, Appl. Phys. Lett. 2005, 86, 112104 , https://doi.org/10.1063/1.1882753.

[91] T. Abe, K. Nagai, S. Kabutomori, M. Kaneko, A. Tajiri, T. Norimatsu, Angew. Chem. Int. Ed. 2006, 45, 2778, https://doi.org/10.1002/anie.200504454.

[92] T. Abe, K. Nagai, T. Ogiwara, S. Ogasawara, M. Kaneko, A Tajiri, T. Norimatsu, J. Electroanal. Chem. 2006, 587, 127, https://doi.org/10.1016/j.jelechem.2005.11.001.

[93] J. Chen, P. Wagner, L. Tong, G. G. Wallace, D. L. Officer, G. F. Swiegers, Angew. Chem. Int. Ed. 2012, 51, 1907, https://doi.org/10.1002/anie.201107355. 
[94] J. Chen, P. Wagner, L. Tong, D. Boskovic, W. Zhang, D. Officer, G. G. Wallace, G. F. Swiegers, Chem. Sci. 2013, 4, 2797, https://doi.org/10.1039/C3SC50812A.

[95] G. Liu, C. Chen, H. Ji, W. Ma, J. Zhao, Sci. China Chem. 2012, 55, 1953 https://doi.org/10.1007/s11426-011-4469-6.

[96] J. T. Kirner, J. J. Stracke, B. A. Gregg, R. G. Finke, ACS Appl. Mater. Interfaces 2014, 6, 13367, https://doi.org/10.1021/am405598w.

[97] P. Bornoz, M. S. Prévot, X. Yu, N. Guijarro, K. Sivula, J. Am. Chem. Soc. 2015, 137, 15338, https://doi.org/10.1021/jacs.5b05724.

[98] M. Alsultan, S. Balakrishnan, J. Choi, R. Jalili, P. Tiwari, P. Wagner, G. F. Swiegers, ACS Appl. Energy Mater. 2018, 1, 4235, https://doi.org/10.1021/acsaem.8b00728.

[99] S.-Y. Park, M. Kim, J. Jung, J. Heo, E. M. Hong, S. M. Choi, J.-Y. Lee, S. Cho, K. Hong, D. C. Lim, J. Power Sources 2017, 341, 411, https://doi.org/10.1016/j.jpowsour.2016.12.017.

[100]J. M. Yu, J. Lee, Y. S. Kim, J. Song, J. Oh, S. M. Lee, M. Jeong, Y. Kim, J. H. Kwak, S. Cho, C. Yang, J.-W. Jang, Nat. Commun. 2020, 11, 5509, https://doi.org/10.1038/s41467-020-19329-0.

[101]T. Abe, H. Ichinohe, S. Kakuta, K. Nagai, Jap. J. Appl. Phys. 2010, 49, 015101, https://doi.org/10.1143/JJAP.49.015101.

[102]X. Fan, T. Wang, B. Gao, H. Gong, H. Xue, H. Guo, L. Song, W. Xia, X. Huang, J. He, Langmuir 2016, 32, 13322, https://doi.org/10.1021/acs.langmuir.6b03107.

[103]Y. Fang, X. Li, X. Wang, ChemSusChem 2019, 12, 2605, https://doi.org/10.1002/cssc.201900291.

[104] Y. Fang, X. Li, Y. Wang, C. Giordano, X. Wang, Appl. Catal. B 2020, 268, 118398, https://doi.org/10.1016/j.apcatb.2019.118398.

[105]N. Karjule, J. Barrio, L. Xing, M. Volokh, M. Shalom, Nano Lett. 2020, 20, 4618, https://doi.org/10.1021/acs.nanolett.0c01484.
[106]J. Liu, H. Wang, Z. P. Chen, H. Moehwald, S. Fiechter, R. van de Krol, L. Wen, L. Jiang, M. Antonietti, Adv. Mater. 2015, 27, 712, https://doi.org/10.1002/adma.201404543.

[107]J. Qin, J. Barrio, G. Peng, J. Tzadikov, L. Abisdris, M. Volokh, M. Shalom, Nat. Commun. 2020, 11, 4701, https://doi.org/10.1038/s41467-020-18535-0.

[108]Y. Yang, S. Wang, Y. Jiao, Z. Wang, M. Xiao, A. Du, Y. Li, J. Wang, L. Wang, Adv. Funct. Mater. 2018, 28, 1805698, https://doi.org/10.1002/adfm.201805698.

[109] M. Yamamoto, L. Wang, F. Li, T. Fukushima, K. Tanaka, L. Sun, H. Imahori, Chem. Sci. 2016, 7, 1430, https://doi.org/10.1039/C5SC03669K.

[110]L. Wang, D. Yan, D. W. Shaffer, X. Ye, B. H. Layne, J. J. Concepcion, M. Liu, C.-Y. Nam, Chem. Mater. 2018, 30, 324, https://doi.org/10.1021/acs.chemmater.7b02889.

[111]C. Dai, X. Gong, X. Zhu, C. Xue, B. Liu, Mater. Chem. Front. 2018, 2, 2021 https://doi.org/10.1039/C8QM00275D.

\section{License and Terms}

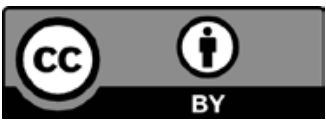

This is an Open Access article under the terms of the Creative Commons Attribution License CC BY 4.0. The material may not be used for commercial purposes.

The license is subject to the CHIMIA terms and conditions: (http:// chimia.ch/component/sppagebuilder/?view = page \&id=12).

The definitive version of this article is the electronic one that can be found at https://doi.org/10.2533/chimia.2021.169 